\title{
Surface accuracy optimization of mechanical parts with multiple circular holes for additive manufacturing based on triangular fuzzy number
}

(C) The Author(s) 2021. This article is published with open access at link.springer.com and journal.hep.com.cn

\begin{abstract}
Surface accuracy directly affects the surface quality and performance of mechanical parts. Circular hole, especially spatial non-planar hole set is the typical feature and working surface of mechanical parts. Compared with traditional machining methods, additive manufacturing (AM) technology can decrease the surface accuracy errors of circular holes during fabrication. However, an accuracy error may still exist on the surface of circular holes fabricated by AM due to the influence of staircase effect. This study proposes a surface accuracy optimization approach for mechanical parts with multiple circular holes for AM based on triangular fuzzy number (TFN). First, the feature lines on the manifold mesh are extracted using the dihedral angle method and normal tensor voting to detect the circular holes. Second, the optimal AM part build orientation is determined using the genetic algorithm to optimize the surface accuracy of the circular holes by minimizing the weighted volumetric error of the part. Third, the corresponding weights of the circular holes are calculated with the TFN analytic hierarchy process in accordance with the surface accuracy requirements. Lastly, an improved adaptive slicing algorithm is utilized to reduce the entire build time while maintaining the forming surface accuracy of the circular holes using digital twins via virtual printing. The effectiveness of the proposed approach is experimentally validated using two
\end{abstract}

Received July 1, 2020; accepted September 6, 2020

Jinghua XU, Hongsheng SHENG, Shuyou ZHANG ( $₫)$, Jianrong TAN State Key Laboratory of Fluid Power and Mechatronic Systems, Zhejiang University, Hangzhou 310027, China; Key Laboratory of Advanced Manufacturing Technology of Zhejiang Province, School of Mechanical Engineering, Zhejiang University, Hangzhou 310027, China E-mail: zsy@zju.edu.cn

Jinlian DENG

Zhejiang Institute of Mechanical and Electrical Engineering, Hangzhou 310053, China mechanical models.

Keywords surface accuracy optimization, multiple circular holes, additive manufacturing (AM), part build orientation, triangular fuzzy number (TFN), digital twins

\section{Introduction}

Circular hole, especially spatial non-planar hole set is the typical feature and working surface of mechanical parts. It is used for the assembly, connection, positioning, and bearing of mechanical parts, but it has many other functions, for instance ventilation and heat dissipation. Traditional machining methods for circular holes include drilling, grinding, reaming, milling, and electrical discharge machining $[1,2]$. However, certain circular holes, such as deep ones, are difficult to manufacture using traditional machining methods due to the limitations in geometry. Moreover, the process parameters of traditional machining methods directly affect the surface accuracy of circular holes, and surface accuracy exerts an important impact on surface quality and part performance $[3,4]$. The surface accuracy errors of traditional machining methods for circular holes include dimension, shape, and position errors.

AM is an advanced freeform fabrication process of producing a 3D physical object by applying a layer-bylayer fabrication process with a series of $2 \mathrm{D}$ layers without the limitation of geometric complexity [5]; it is also known as rapid prototyping (RP) and 3D printing. Many types of AM technology, such as fused deposition modeling (FDM), stereo lithography apparatus, selective laser sintering and selective laser melting, are used in various fields [6]. The layer-by-layer fabrication process generates a staircase effect (SE) on the surface of parts fabricated by $\mathrm{AM}$ [7]. SE results in a volumetric error (VE), which is the 
difference between the volume of the material used for the fabricated part and the volume specified by the CAD model [8]. SE adversely affects the surface accuracy of parts fabricated by AM.

Two crucial factors, namely, part build orientation and slicing algorithm, influence the surface accuracy of parts fabricated by AM. Part build orientation directly affects the surface accuracy, support structure, and build time of parts fabricated by AM. The VE, support structure, and build time of such parts vary with the change in part build orientation, resulting in different surface accuracies. The slicing algorithm also exerts an important impact on the surface accuracy and build time of parts fabricated by AM. A small layer thickness equates to high surface accuracy and long build time. Conversely, a large layer thickness leads to a short build time and low surface accuracy.

Various approaches have been proposed for the determination of part build orientation. Rattanawong et al. [8] developed an approach to determine the part build orientation in RP by considering the VE encountered in parts during the building process, which assumes that a complex part is to be constructed from a combination of basic primitive volumes. Byun and Lee [9] presented a method to determine the optimal part orientation by using a genetic algorithm (GA) and considering part surface roughness and build time. Zhao [10] selected the optimal build orientation by adopting a multi-objective optimization algorithm based on satisfactory degree theory, which includes SE, area of supports, and production time. Luo and Wang [11] proposed a fast determining scheme of the optimal slicing orientation with the least VE to reduce the inevitable VE induced in the phase of model slicing, which influences the shape accuracy of the fabricated entity. Ezair et al. [12] presented a method to optimize the support structure volume of 3D models on the basis of determining the orientation of models. Pereira et al. [13] proposed a strategy to optimize SE and support generation with global derivative-free optimization for symmetrical objects; the strategy can determine the optimal part build orientation in AM.

Slicing algorithms can be classified into uniform and adaptive slicing depending on whether the layer thickness changes. Uniform and adaptive slicing are utilized for the original CAD model and the tessellated CAD model. Uniform slicing, which slices the model with a set of horizontal slicing planes with the same layer thickness, has been widely adopted for all kinds of AM technology. Miyanaji et al. [14] researched green part printing process for 3D printing. Shan et al. [15] studied the print forming method and device for multi-material casting of mechanical parts. Zhang and Joshi [16] developed an improved and robust slicing algorithm with efficient contour construction by using a special linked list structure. This algorithm can identify outer and inner contours automatically. Zeng et al. [17] and Qi et al. [18] presented the slicing algorithms for complex constructive solid geometry models. Their algorithms achieve a compromise between slicing accuracy and time on the basis of the adaptive layer depth normal image.

Adaptive slicing slices the model into variable layer thicknesses as determined by the geometric change in the model along the build direction. It aims to reduce build time while maintaining surface quality. Dolenc and Makela [7] proposed the cusp height concept for evaluating SE that is used to determine variable layer thicknesses. They utilized the adaptive slicing algorithm with handling peaks and flat areas of the STL model. Kulkarni and Dutta [19] developed an adaptive slicing method to determine layer thickness by calculating the normal curvature of the surface in the vertical direction. However, their method is limited to parametric algebraic surfaces. The fractographic mechanism of hole and its effect on the part quality are investigated in Refs. [20,21]. Rianmora and Koomsap [22] presented an adaptive direct slicing method that applies image processing to determine the appropriate thickness for each sliced layer and recommend slicing positions on a 3D CAD model. Hayasi and Asiabanpour [23] proposed a new adaptive slicing approach to cut a 3D solid model at the predefined sheets' thickness for the fully dense freeform fabrication process. In their approach, all internal and external features are investigated to reduce the part geometry deviation. Butt et al. [24] produced customised electronic circuitry with dual extruder 3D printer.

Although AM can reduce the surface accuracy errors of circular holes during fabrication compared with traditional machining methods, such errors may still exist in circular holes fabricated by AM because of SE. The selection of the part build orientation and slicing algorithm is crucial in improving the surface accuracy of the circular holes of a part fabricated by AM because the SE of a part fabricated by AM is generally difficult to be eliminated. Therefore, the circular hole feature of a part's STL model must be determined.

STL is a mesh model and the commonly used file format for AM; it is composed of a set of unordered discrete triangular facets [5]. The mesh model's feature lines that represent crucial characteristics must be determined and can be utilized in mesh simplification, surface reconstruction, and mesh segmentation. Ohtake et al. [25] proposed a method to detect the ridge and valley of a surface by estimating the high-order partial derivative of the surface. Kim and Kim [26] utilized an algorithm to estimate the local differential information of the mesh by using the moving least squares, and the zero-crossing point of the curvature partial derivative was adopted as the feature point. Sunil and Pande [27] proposed a method of feature extraction based on the surface normal vector. The method analyzes the angle between two faces sharing an edge or the difference between the current point and the normal direction of its one-ring neighborhood point. Shimizu et al. [28] introduced a mesh smoothing method that recognizes features by using the normal tensor voting technique. Kim 
et al. [29] presented a feature line recognition method that uses the normal tensor voting technique to estimate the curvature in large-scale and noisy grids. Jiao and Bayyana [30] identified the feature points, feature edges, and directions of feature edges by means of the dihedral angle, edge angle, and feature vector of the vertex normal tensor matrix, which was extended to the case of $\mathrm{C}^{2}$ discontinuous mesh edges. Qu and Stucker [31] developed a method to recognize circular holes from 3D models in the STL format for generating a toolpath in drilling holes; the method was then utilized for post machining in AM. Yang et al. [32] presented a method to evaluate differential quantities at the vertices of triangular meshes that may approximate potential piecewise smooth surfaces with discontinuous normals or curvatures at the joint lines. Tong and Tai [33] proposed a unified variational framework for detecting generic feature lines on polygonal meshes and demonstrated the framework's practical implementation.

The current work attempts to optimize the surface accuracy of the circular holes of mechanical parts fabricated by AM by using an optimal part build orientation and an appropriate slicing algorithm. The circular holes of a part's STL model are detected. The optimal part build orientation is determined by minimizing the VE of all circular holes to optimize the surface accuracy of the circular holes. Given the different surface accuracy requirements of the circular holes of mechanical parts, different weights are assigned to the corresponding circular holes in the calculation of the optimal part build orientation. The selection of optimization approaches is essential because the determination of the optimal part build orientation is a time-consuming process. Many optimization approaches, for example GA, particle swarm optimization, multiphase level set method [34], and artificial neural network method [35], are used in various fields. GA is adopted to solve the optimal part build orientation due to its efficiency. Moreover, the proposed adaptive slicing algorithm in Ref. [7] is improved to reduce the overall build time while maintaining the forming surface accuracy of the circular holes. This work is an extension of previous work [36-38].

The layout of this paper is as follows. The method of detecting the circular hole feature is described in Section 2. Section 3 presents the determination algorithm of part build orientation for optimizing the surface accuracy of circular holes. An improved adaptive slicing approach is introduced in Section 4. Two mechanical models are used to verify the proposed method, and a discussion of the corresponding results is presented in Section 5. Section 6 provides the conclusions.

\section{Circular hole feature detection of a part's STL model}

The feature lines should be extracted from the STL model before detecting the circular hole feature. The proposed feature line extraction methods are advantageous for several models; however, no single method is suitable for all kinds of models because models are diverse. The dihedral angle method [27] and normal tensor voting theory $[28,29]$ are utilized to extract the feature lines of a part's STL model. First, the explicit and implicit feature edges are extracted with the dihedral angle method. Second, normal tensor voting theory is utilized to determine if the implicit feature edge is a feature edge. The feature edge is a line segment with a finite length composed of two vertices; it can also be called a feature line. The intersection of the circular hole feature and the surface of the STL model is a closed line loop. On this basis, the circular hole feature can be detected using the extracted feature lines [31].

\subsection{Initial feature edge extraction}

Dihedral angle is a scale-independent measurement with high feature discrimination capability and stability [27]. Assume that $e$ is an edge shared by two adjacent triangular facets, namely, $f_{1}$ and $f_{2}$, and $\boldsymbol{n}_{1}$ and $\boldsymbol{n}_{2}$ are the unit normal vectors of $f_{1}$ and $f_{2}$, respectively. The dihedral angle $\theta_{\mathrm{e}}$ (in ${ }^{\circ}$ ) defined in edge $e$ is the angle between $\boldsymbol{n}_{1}$ and $\boldsymbol{n}_{2}$ and can be expressed as

$$
\theta_{\mathrm{e}}=\arccos \frac{\boldsymbol{n}_{1} \cdot \boldsymbol{n}_{2}}{\left|\boldsymbol{n}_{1}\right| \cdot\left|\boldsymbol{n}_{2}\right|} .
$$

If $\theta_{\mathrm{e}}$ is larger than a user-specified threshold $\theta_{\mathrm{t} 1}$ (in ${ }^{\circ}$ ), i.e., $\theta_{\mathrm{e}} \in\left[\theta_{\mathrm{t} 1}, 180^{\circ}\right)$, then $e$ is identified as a feature edge, i.e., explicit feature edge. However, a suitable threshold $\theta_{\mathrm{t} 1}$ is difficult to select because models vary. If $\theta_{\mathrm{t} 1}$ is too large, several implicit feature edges will be discarded; if $\theta_{\mathrm{t} 1}$ is too small, certain edges will be incorrectly identified as implicit feature edges. A common approach is to use the average value $\bar{\theta}_{\mathrm{e}}$ of all dihedral angles as the initial threshold, which is given by

$$
\left\{\begin{array}{l}
\theta_{\mathrm{t} 1}=\bar{\theta}_{\mathrm{e}}+\mu_{1} \sigma_{\theta_{\mathrm{e}}}, \quad \bar{\theta}_{\mathrm{e}}<20, \\
\theta_{\mathrm{t} 1}=\bar{\theta}_{\mathrm{e}}, \quad \bar{\theta}_{\mathrm{e}} \geqslant 20,
\end{array}\right.
$$

where $\sigma_{\theta_{\mathrm{e}}}$ is the standard value of all dihedral angles and $\mu_{1} \in(0,1)$ is a weight value that is proportional to mesh accuracy.

Explicit feature edges can be obtained by $\theta_{\mathrm{t} 1}$, but the implicit feature edges located at the boundary of the transition feature surface, such as the edges between the transition fillet and the surface of the STL model, may be ignored. Therefore, another threshold $\theta_{\mathrm{t} 2}\left(\right.$ in $\left.^{\circ}\right)$ is used to identify several implicit feature edges.

$$
\theta_{\mathrm{t} 2}=\frac{\sum_{i} \theta_{i}^{\mu_{2}}}{n_{\mathrm{e}}},
$$

where $\theta_{i}^{\mu_{2}}$ is the $i$ th dihedral angle that meets 
$\theta_{i}^{\mu_{2}} \in\left(0, \mu_{2} \bar{\theta}_{\mathrm{e}}\right], \mu_{2} \in(0,1)$ is a weight value that is proportional to mesh accuracy, and $n_{\mathrm{e}}$ is the number of edges that meet the requirement.

When $\theta_{\mathrm{e}} \in\left(0, \theta_{t 2}\right]$, the corresponding edge $e$ is identified as an implicit feature edge. All explicit feature edges are identified as feature edges. Whether an implicit feature edge is a feature edge requires further judgment.

\subsection{Second feature edge extraction}

Normal tensor voting theory [28,29] is implemented to determine whether an implicit feature edge is a feature edge after initial feature edge extraction. The normal voting tensor of a vertex $v$ on a triangular mesh is defined using the unit normal vectors of neighbor triangular facets (shown in Fig. 1).

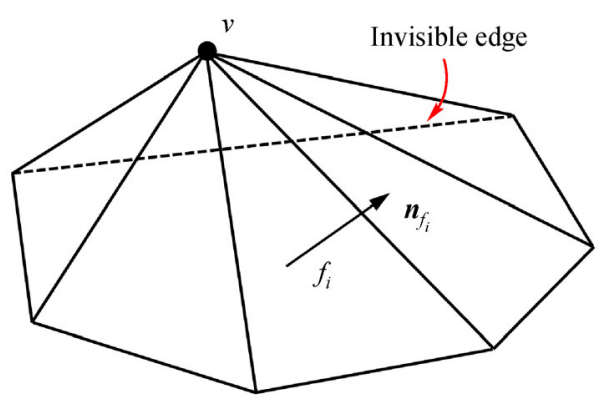

Fig. 1 One-ring neighbor triangular facets of vertex $v$ in the manifold mesh.

$\boldsymbol{V}_{\mathrm{v}}^{f_{i}}$ is a covariance matrix of triangular facet $f_{i}$, which is defined by the unit normal vector $\boldsymbol{n}_{f_{i}}=(a, b, c)$ of $f_{i}$. $\boldsymbol{V}_{\mathrm{v}}^{f_{i}}$ can be expressed as

$$
\boldsymbol{V}_{\mathrm{v}}^{f_{i}}=\boldsymbol{n}_{f_{i}} \cdot \boldsymbol{n}_{f_{i}}^{\mathrm{T}}=\left(\begin{array}{ccc}
a^{2} & a b & a c \\
a b & b^{2} & b c \\
a c & b c & c^{2}
\end{array}\right) .
$$

The normal voting tensor $\boldsymbol{T}_{\mathrm{v}}$ of vertex $v$ is the sum of the weighted covariance matrices of its neighbor triangular facets, which can be expressed as

$$
\boldsymbol{T}_{\mathrm{v}}=\sum_{f_{i} \in N_{\mathrm{f}}(v)} \mu_{f_{i}} \boldsymbol{V}_{\mathrm{v}}^{f_{i}}
$$

where $N_{\mathrm{f}}(v)$ is the set of all neighbor triangular facets of vertex $v$ and $\mu_{f_{i}}$ is a weight expressed as

$$
\mu_{f_{i}}=\frac{\operatorname{area}\left(f_{i}\right)}{\operatorname{area}(\max )} \cdot \exp \left(-\frac{\left\|\boldsymbol{c}_{f_{i}}-\boldsymbol{P}_{\mathrm{v}}\right\|}{\sigma / 3}\right),
$$

where $\operatorname{area}\left(f_{i}\right)$ is the area of $f_{i}$, area $(\max )$ is the maximum area of $N_{\mathrm{f}}(v), \boldsymbol{c}_{f_{i}}$ is the barycenter of $f_{i}, \boldsymbol{P}_{\mathrm{v}}$ is the position of vertex $v$, and $\sigma$ is the edge length of a cube that defines the neighboring space of each vertex.
$\boldsymbol{T}_{\mathrm{v}}$ is a symmetric positive semi-definite matrix and can be presented as

$$
\boldsymbol{T}_{\mathrm{v}}=\lambda_{1} \boldsymbol{e}_{1} \boldsymbol{e}_{1}^{\mathrm{T}}+\lambda_{2} \boldsymbol{e}_{2} \boldsymbol{e}_{2}^{\mathrm{T}}+\lambda_{3} \boldsymbol{e}_{3} \boldsymbol{e}_{3}^{\mathrm{T}},
$$

where $\lambda_{1} \geqslant \lambda_{2} \geqslant \lambda_{3} \geqslant 0, \lambda_{1}, \lambda_{2}$, and $\lambda_{3}$ are eigenvalues, and $\boldsymbol{e}_{1}, \boldsymbol{e}_{2}$, and $\boldsymbol{e}_{3}$ are the corresponding unit eigenvectors of $\lambda_{1}$, $\lambda_{2}$, and $\lambda_{3}$, respectively. $\boldsymbol{e}_{1}$ represents the approximation of the surface normal vector, and $\boldsymbol{e}_{2}$ and $\boldsymbol{e}_{3}$ are the principal curvatures.

Figure 2 shows the eigenvalue distribution of normal tensor voting for corresponding features. The vertices of the triangular facet can be classified into face, sharp edge, and corner according to the eigenvalues as follows [28]:

Face: $\lambda_{1}$ is dominant, and $\lambda_{2}$ and $\lambda_{3}$ are close to 0 ;

Sharp edge: $\lambda_{1}$ and $\lambda_{2}$ are dominant, and $\lambda_{3}$ is close to 0 ;

Corner: $\lambda_{1}, \lambda_{2}$, and $\lambda_{3}$ are approximately equal.

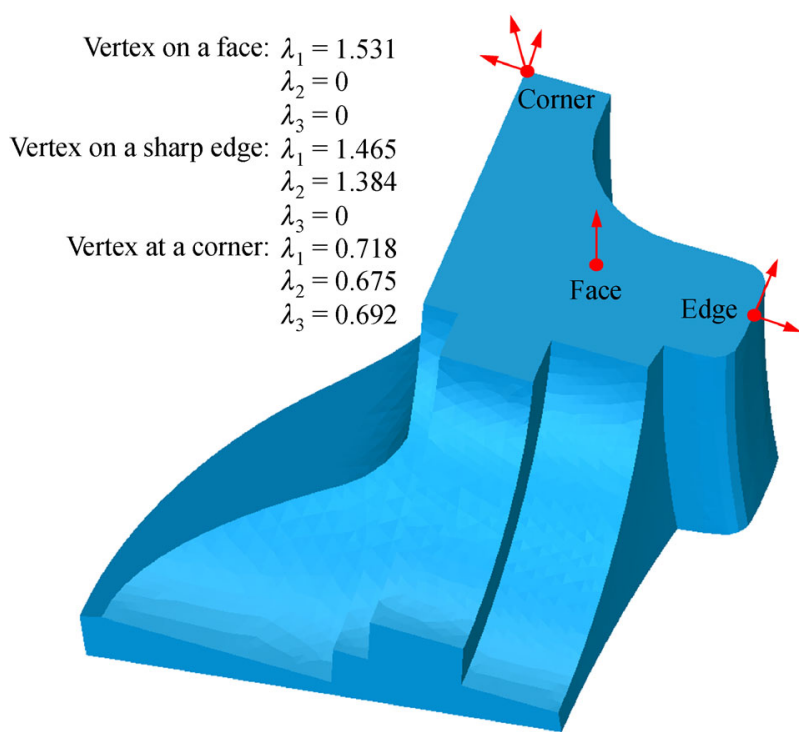

Fig. 2 Eigenvalues of normal tensor voting for corresponding features.

Sharp edges and corner vertices are called feature vertices. Therefore, the key point of determining whether an implicit feature edge is a feature edge is to determine whether the two end vertices $\left(P_{\mathrm{ev} 1}\right.$ and $\left.P_{\mathrm{ev} 2}\right)$ of the implicit feature edge are feature vertices or not by using normal tensor voting. The implicit feature edge is not a feature edge when $P_{\mathrm{ev} 1}$ and $P_{\mathrm{ev} 2}$ are not feature vertices. Any feature edge located at the transition feature has at least two adjacent connected feature edges because the transition feature is located at the joint of two smooth surfaces. Therefore, if an implicit feature edge with two feature vertices only has one adjacent feature edge, then this implicit feature edge is not a feature edge; otherwise, this implicit feature edge is a feature edge. The feature lines of the STL model can be constructed by connecting the feature vertices and feature edges based on their adjacent 
topology relationship.

As shown in Fig. 3, the circular hole may be located on a plane or curved surface. The feature edges of the circular hole located on a curved surface are explicit feature edges that can be extracted by the dihedral angle method. However, the feature edges of the circular hole located on a plane are extracted by using normal tensor voting because they are implicit feature edges located on the transition fillet. The intersection of the circular hole and the surface of the STL model is a closed line loop. Therefore, the circular hole can be detected based on the closed line loops after extracting the feature lines of the part's STL model. The approach proposed in Ref. [31] is utilized to detect the circular hole and obtain circular hole information, including axis direction, depth, and diameter, by using the closed line loops. The triangular facet sets of the detected circular holes are depicted as $\mathrm{CH}_{1}, \mathrm{CH}_{2}, \ldots, \mathrm{CH}_{n}$, which are shown in Fig. 3(b).

\section{Determination of part build orientation based on weighted VE}

\subsection{Calculation of weighted VE}

For any AM fabricated 3D part, the part model is sliced into a set of 2D layers by a series of defined horizontal slicing planes with different layer thicknesses. Figure 4 illustrates SE during AM fabrication that causes VE. Here, the build direction is $\boldsymbol{Z}=(0,0,1)^{\mathrm{T}}$ along the vertical direction, $\boldsymbol{n}_{\mathrm{f}}$ is the unit normal vector of an inclined facet $f$, $d$ is the layer thickness, $d_{\mathrm{t}}$ is the length between two adjacent layers along the horizontal direction, and $\alpha$ (in ${ }^{\circ}$ ) is the angle between $\boldsymbol{Z}$ and $\boldsymbol{n}_{\mathrm{f}}$, i.e., $\alpha \in\left[0^{\circ}, 180^{\circ}\right] . \delta$ is the cusp height [7], which is the distance between a triangular facet of the original STL model and the actual formed surface of a part.

Cusp height $\delta$ is given by

$$
\delta=d|\cos \alpha|
$$

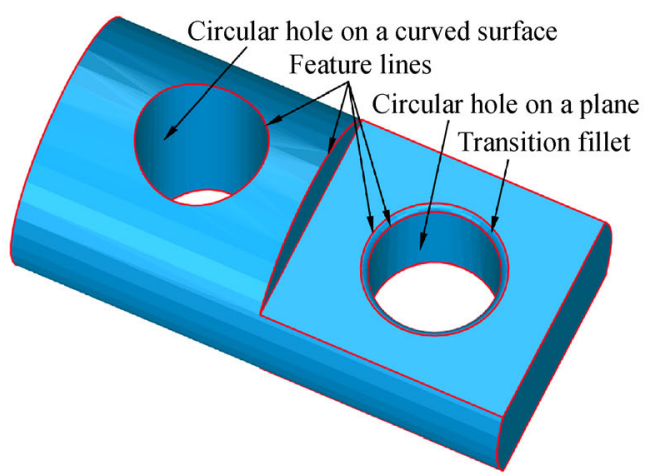

(a)
The following two equations can be obtained from Fig. 4:

$$
\begin{aligned}
& \cos \alpha=\frac{\left|\boldsymbol{Z} \cdot \boldsymbol{n}_{\mathrm{f}}\right|}{|\boldsymbol{Z}| \cdot\left|\boldsymbol{n}_{\mathrm{f}}\right|}, \\
& \sin \alpha=\frac{\left|\boldsymbol{Z} \times \boldsymbol{n}_{\mathrm{f}}\right|}{|\boldsymbol{Z}| \cdot\left|\boldsymbol{n}_{\mathrm{f}}\right|} .
\end{aligned}
$$

The area error, $\Delta A$, between the actual formed surface and theoretical outer surface of the local micro area of one layer is expressed as

$$
\Delta A=\frac{1}{2} d d_{\mathrm{t}}=\frac{d^{2}}{2 \tan \alpha}=\frac{d^{2}\left|\boldsymbol{Z} \cdot \boldsymbol{n}_{\mathrm{f}}\right|}{2\left|\boldsymbol{Z} \times \boldsymbol{n}_{\mathrm{f}}\right|} .
$$

As shown in Fig. 5, $l_{i j}$ is the length of the average intersecting line segment of the $i$ th layer and the $j$ th triangular facet $\left(f_{j}\right)$, and $d A_{j}$ is the area of $f_{j}$ lays in the $i$ th layer. The $\operatorname{VE}$ of $f_{j}$ lays in the $i$ th layer is written as

$$
\Delta V E=\Delta A l_{i j}=\frac{d^{2}\left|\boldsymbol{Z} \cdot \boldsymbol{n}_{\mathrm{f}}\right|}{2\left|\boldsymbol{Z} \times \boldsymbol{n}_{\mathrm{f}}\right|} l_{i j} .
$$

Therefore, the VE of $f_{j}$ can be regarded as the sum of all $\Delta V E$ existing in $f_{j}$ and is expressed as

$$
V E_{j}=\sum_{i} \Delta V E=\sum_{i} \frac{d^{2}\left|\boldsymbol{Z} \cdot \boldsymbol{n}_{\mathrm{f}}\right|}{2\left|\boldsymbol{Z} \times \boldsymbol{n}_{\mathrm{f}}\right|} l_{i j} .
$$

Given that the magnitude of the normal vector $\boldsymbol{n}_{f_{j}}$ of $f_{j}$ is $1, d A_{j}$ can be written as

$$
d A_{j}=\frac{d l_{i j}}{\sin \alpha}=\frac{d l_{i j}}{\left|\boldsymbol{Z} \times \boldsymbol{n}_{f_{j}}\right|} .
$$

Equation (13) can be expressed as

$$
V E_{j}=\sum_{i} \frac{d\left|\boldsymbol{Z} \cdot \boldsymbol{n}_{f_{j}}\right|}{2} d A_{j}=\frac{d\left|\boldsymbol{Z} \cdot \boldsymbol{n}_{f_{j}}\right|}{2} A_{j}, j \in\left[1, n_{\mathrm{f}}\right],
$$

where $A_{j}$ is the area of $f_{j}$ and $n_{\mathrm{f}}$ is the number of triangular facets of the part model. Hence, the whole VE of the part

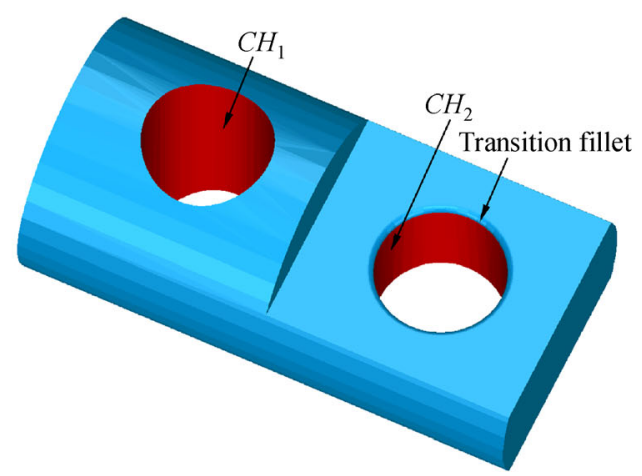

(b)

Fig. 3 Circular hole features on a plane and curved surface. (a) Feature lines of the STL model; (b) triangular facet sets of circular holes. 


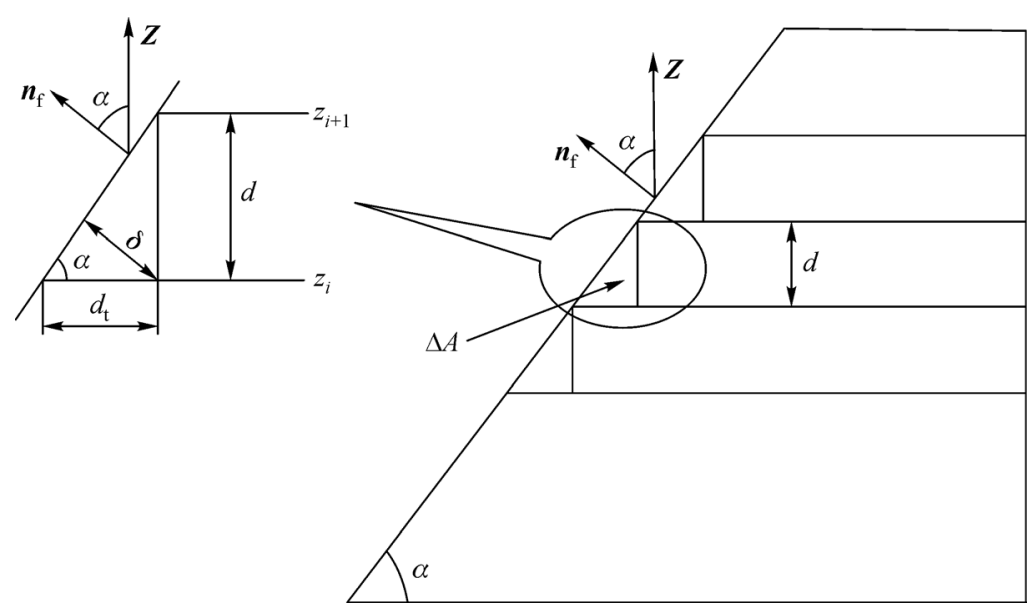

Fig. 4 Staircase effect in the additive manufacturing fabrication process.

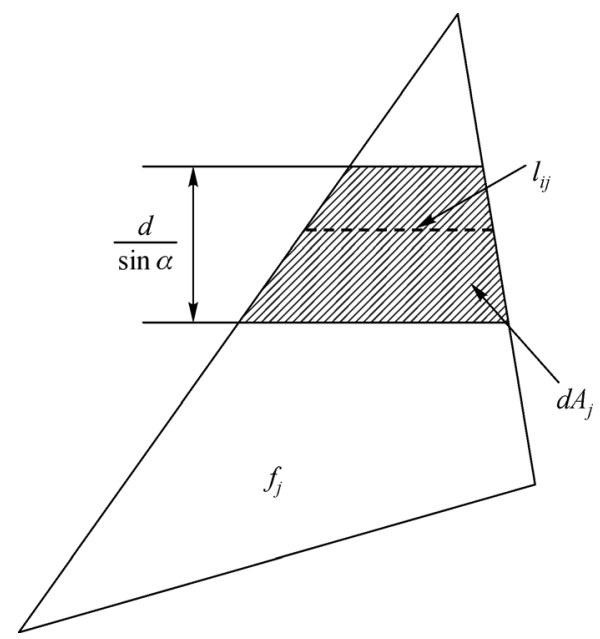

Fig. 5 Staircase effect in one triangular facet.

can be written as

$$
V E=\sum_{j=1}^{n_{\mathrm{f}}} V E_{j}=\sum_{j=1}^{n_{\mathrm{f}}} \frac{d\left|\boldsymbol{Z} \cdot \boldsymbol{n}_{f_{j}}\right|}{2} A_{j} .
$$

The surface accuracy of a part can be optimized by reducing VE, that is, by minimizing the entire VE of the part, which can be expressed by the function $f(\boldsymbol{Z})$.

$$
f(\boldsymbol{Z})=\min \sum_{j=1}^{n_{\mathrm{f}}} \frac{d \cdot\left|\boldsymbol{Z} \cdot \boldsymbol{n}_{f_{j}}\right|}{2} A_{j} .
$$

The function $f(\boldsymbol{Z})$ can be regarded as an optimization problem that aims to determine an optimal part build direction to minimize the entire VE of a part. The build direction remains to be $\boldsymbol{Z}=(0,0,1)^{\mathrm{T}}$. Thus, the optimization problem of minimizing the entire VE of a part is transformed into a problem of finding an optimal part build orientation, which means making the part model rotate around the $X$ - and $Y$-axis at a certain angle. The part model does not need to rotate around the $Z$-axis because it does not affect the manufacturing process. The rotation transformation matrix of the part model after rotating at angles of $\theta_{x}\left(\right.$ in $\left.^{\circ}\right)$ and $\theta_{y}\left(\right.$ in $\left.^{\circ}\right)$ about the $X$ - and $Y$-axis is written as

$$
\boldsymbol{T r}=\boldsymbol{R}_{y} \cdot \boldsymbol{R}_{x},
$$

where $\boldsymbol{R}_{x}$ and $\boldsymbol{R}_{y}$ are the rotation matrices around the $X$ - and $Y$-axis given by Eqs. (19) and (20), respectively.

$$
\begin{gathered}
\boldsymbol{R}_{x}=\left[\begin{array}{ccc}
1 & 0 & 0 \\
0 & \cos \theta_{x} & -\sin \theta_{x} \\
0 & \sin \theta_{x} & \cos \theta_{x}
\end{array}\right], \\
\boldsymbol{R}_{y}=\left[\begin{array}{ccc}
\cos \theta_{y} & 0 & \sin \theta_{y} \\
0 & 1 & 0 \\
-\sin \theta_{y} & 0 & \cos \theta_{y}
\end{array}\right] .
\end{gathered}
$$

The new normal vector $\boldsymbol{n}_{t f_{j}}$ of $f_{j}$ after rotation transformation is expressed as

$$
\boldsymbol{n}_{\mathrm{t} f_{j}}=\boldsymbol{T r} \cdot \boldsymbol{n}_{f_{j}}=\boldsymbol{R}_{y} \cdot \boldsymbol{R}_{x} \cdot \boldsymbol{n}_{f_{j}} .
$$

The entire VE of a part can be optimized by using an optimal part build orientation. However, the surface accuracy of the circular holes of a part is the focus. Therefore, the VE of all circular holes of a part must be optimized. The best build orientation of a circular hole is the orientation whose axis direction is parallel to the AM build direction. The unit normal vectors of all triangular facets of the circular hole are perpendicular to the AM build direction at that orientation. Therefore, the cusp heights of the circular hole are zero according to Eq. (8), and the corresponding VE of the circular hole is also zero according to Eq. (16) at that orientation. However, if several circular holes have axis directions that are not parallel to each other, then an optimal build orientation 
must be selected for all circular holes.

Generally, the surface accuracy requirements of the different circular holes of mechanical parts vary, and different weights should be assigned to the circular holes during calculation. The optimal part build orientation is the orientation that minimizes the weighted volumetric error (WVE) of a part, and it is expressed as

$$
\begin{array}{r}
\min f\left(\theta_{x}, \theta_{y}\right)=\min \left\{\sigma _ { 1 } \left(\omega_{1} \sum_{f_{j} \in C H_{1}} \frac{d\left|\boldsymbol{Z} \cdot \boldsymbol{n}_{f_{j}}\right|}{2} A_{j}+\cdots\right.\right. \\
\left.\left.\quad+\omega_{i} \sum_{f_{j} \in C H_{i}} \frac{d\left|\boldsymbol{Z} \cdot \boldsymbol{n}_{f_{j}}\right|}{2} A_{j}\right)+\sigma_{2} \sum_{f_{j} \in R M} \frac{d\left|\boldsymbol{Z} \cdot \boldsymbol{n}_{f_{j}}\right|}{2} A_{j}\right\},
\end{array}
$$

where $\omega_{i}$ is the weight of the $i$ th circular hole $\left(\omega_{i} \geqslant 0\right)$ and $\sum_{i} \omega_{i}=1 . \sigma_{1}$ is the weight of all circular holes, and $\sigma_{2}$ is the weight of the remaining part of the part model, except for all circular holes. If $\sigma_{1}=0.8$ then $\sigma_{2}=1-\sigma_{1} . R M$ represents the triangular facet set of the remaining part of the part model, except for all circular holes. The rotation range of $\theta_{x}$ and $\theta_{y}$ is $\left[0^{\circ}, 180^{\circ}\right]$, which includes all possible orientations of a part.

The solution $\left(\theta_{x}, \theta_{y}\right)$ and function value of Eq. (22) are calculated using GA. The optimization problem of Eq. (22) can be expressed as

$$
\left\{\begin{array}{l}
\min f\left(\theta_{x}, \theta_{y}\right), \\
\text { s.t. } 0^{\circ} \leqslant \theta_{x} \leqslant 180^{\circ}, \\
0^{\circ} \leqslant \theta_{y} \leqslant 180^{\circ} .
\end{array}\right.
$$

A population size of 50 and maximum number of generations of 200 are considered the stopping criteria of GA. The probabilities of crossover and mutation are 0.8 and 0.02 , respectively.

3.2 Determination of the corresponding weights for circular holes

The weights of criteria are usually defined by clear values given by the user. However, such clear values cannot be obtained easily because human judgment is often vague. Fuzzy evaluation, which is an implementation of fuzzy mathematics, is a highly intuitive and feasible method of determining criteria weights. Fuzzy mathematics is widely used in fuzzy decision-making [39], fuzzy control [40], and other fields. Weight $\omega_{i}$ is determined via the analytic hierarchy process (AHP) [41] on the basis of fuzzy evaluation by using linguistic variables of the relative importance of the surface accuracy requirements for different circular holes. The linguistic variables are expressed as triangular fuzzy number (TFN) [42,43], and each circular hole is regarded as a criterion.
The membership function of a TFN $M_{i}=\left(a_{i}, b_{i}, c_{i}\right)$ is presented as

$$
\mu_{M}(x)= \begin{cases}\frac{x-a_{i}}{b_{i}-a_{i}}, & a_{i} \leqslant x \leqslant b_{i}, \\ \frac{x-c_{i}}{b_{i}-c_{i}}, & b_{i} \leqslant x \leqslant c_{i}, \\ 0, & \text { otherwise }\end{cases}
$$

where $0<a_{i} \leqslant b_{i} \leqslant c_{i}, a_{i}$ and $c_{i}$ are the lower and upper bounds of $M_{i}$, respectively, and $b_{i}$ is the median of $M_{i}$, i.e., $\mu_{M}\left(b_{i}\right)=1 . s_{i}=c_{i}-a_{i}$ represents the fuzziness of $M_{i}$. The larger $s_{i}$ is, the fuzzier $M_{i}$ is. The reciprocal of $M_{i}$ is written as

$$
M_{i}^{-1}=\frac{1}{M_{i}}=\left(\frac{1}{c_{i}}, \frac{1}{b_{i}}, \frac{1}{a_{i}}\right)
$$

A corresponding non-fuzzy number exists for $M_{i}$ and is presented as

$$
S\left(M_{i}\right)=\frac{a_{i}+2 b_{i}+c_{i}}{4} .
$$

The linguistic values of the fuzzy evaluation of the relative importance of two criteria and their corresponding TFN are shown as follows:

Equal importance: $M_{1}=(1,1,1)$;

Intermediate values between $M_{1}$ and $M_{3}: M_{2}=(1,2,3)$;

Moderate importance: $M_{3}=(2,3,4)$;

Intermediate values between $M_{3}$ and $M_{5}: M_{4}=(3,4,5)$;

Essential importance: $M_{5}=(4,5,6)$;

Intermediate values between $M_{5}$ and $M_{7}: M_{6}=(5,6,7)$;

High importance: $M_{7}=(6,7,8)$;

Intermediate values between $M_{7}$ and $M_{9}: M_{8}=(7,8,9)$;

Extreme importance: $M_{9}=(8,9,10)$.

For example, if the importance of criteria $u_{i}$ relative to criteria $u_{j}$ expressed by TFN $q_{i j}=\left(a_{i j}, b_{i j}, c_{i j}\right)$ is moderate, then $q_{i j}=M_{3}=(2,3,4)$. The corresponding importance of criteria $u_{j}$ relative to criteria $u_{i}$ is $q_{j i}=\frac{1}{q_{i j}}=$ $\left(\frac{1}{4}, \frac{1}{3}, \frac{1}{2}\right)$.

To obtain weight $\omega_{i}$, the TFN fuzzy judgment matrix $\boldsymbol{Q}=\left(q_{i j}\right)_{n \times n}$ is established by a pairwise comparison of the surface accuracy requirements in all circular holes. $q_{i j}=$ $\left(a_{i j}, b_{i j}, c_{i j}\right)$ is the corresponding TFN whose value is shown as previously mentioned. Next, $q_{i j}$ is transformed into a non-fuzzy number $r_{i j}^{\prime}=S\left(q_{i j}\right)=\frac{a_{i j}+2 b_{i j}+c_{i j}}{4}$, which can construct a non-fuzzy judgment matrix $\boldsymbol{R}^{\prime}=\left(r_{i j}^{\prime}\right)_{n \times n}$. If $r_{i j}^{\prime} \cdot r_{j i}^{\prime} \neq 1$, then $\boldsymbol{R}^{\prime}$ is not a reciprocal matrix and needs to be adjusted into a reciprocal matrix $\boldsymbol{R}=\left(r_{i j}\right)_{n \times n}$ by using Eq. (27): 


$$
r_{i j}=\frac{r_{i j}^{\prime}}{\sqrt{r_{i j}^{\prime} \cdot r_{j i}^{\prime}}} .
$$

If matrix $\boldsymbol{R}$ passes the AHP consistency test, then the weight $\omega_{i}$ of each circular hole can be obtained with the AHP method; otherwise, $\boldsymbol{R}$ needs to be adjusted.

$$
C R=\frac{C I}{R I},
$$

where $C R$ is the random consistency ratio, $R I$ is the random consistency index whose values are given in Table 1, and $C I$ is the consistency index. $C I$ is expressed as

$$
C I=\frac{\lambda_{\max }-n}{n-1},
$$

where $\lambda_{\max }$ is the maximum eigenvalue of matrix $\boldsymbol{R}$ and $n$ is the order of matrix $\boldsymbol{R}$.

Table 1 Standard values of $R I$

\begin{tabular}{ll}
\hline$n$ & $R I$ \\
\hline 1 & 0.00 \\
2 & 0.00 \\
3 & 0.58 \\
4 & 0.90 \\
5 & 1.12 \\
6 & 1.24 \\
7 & 1.32 \\
8 & 1.41 \\
9 & 1.45 \\
10 & 1.49 \\
\hline
\end{tabular}

When $C R<0.10$, matrix $\boldsymbol{R}$ passes the AHP consistency test. Thus, the elements of matrix $\boldsymbol{R}$ are normalized by column, and the resulting matrix is added in rows to obtain a column vector. The corresponding weight vector $\boldsymbol{W}=$ $\left(\omega_{1}, \omega_{2}, \ldots, \omega_{i}\right)^{\mathrm{T}}$ of all circular holes of a part can be obtained by normalizing the column vector. This approach can be applied to various mechanical parts with multiple circular holes with different surface accuracy requirements. Then, the solution $\left(\theta_{x}, \theta_{y}\right)$ and function value of the optimization problem of Eq. (23) can be calculated.

\section{Adaptive slicing}

The surface accuracy of the circular holes of a part is the focus. The adaptive slicing method proposed by Ref. [7] is improved to slice a part and reduce the build time while maintaining the forming surface accuracy of the circular holes. The maximum and minimum $z$ coordinates at the optimal orientation of each circular hole of a part denoted as $z_{C H_{i} \text {,max }}$ and $z_{C H_{i}, \text { min }}$, respectively, are determined. The aim is to preserve the integrity of the circular hole feature while slicing a part. The maximum and minimum allowable layer thicknesses are denoted by $d_{\max }$ and $d_{\text {min }}$, respectively, and they are limited by actual AM technology.

The height of the initial slicing plane of $\mathrm{CH}_{i}$ is $z_{i, 1}=z_{C H_{i} \text { min }}$. The height of the $j$ th slicing plane of $\mathrm{CH}_{i}$ is expressed as

$$
z_{i, j}=z_{i, j-1}+d_{C H_{i},-1},
$$

where $z_{i, j} \in\left[z_{C H_{i}, \min }, z_{C H_{i}, \max }\right), j \geqslant 2$, and $d_{C H_{i},}$ is the $j$ th layer thickness of $\mathrm{CH}_{i}$. The intersected triangular facets of the $j$ th slicing plane of $\mathrm{CH}_{i}$ can be obtained by comparing the positional relationship of the $z$ coordinate between the slicing plane and the triangular facets of $\mathrm{CH}_{i}$.

Therefore, the layer thicknesses of $\mathrm{CH}_{i}$ that meet the surface accuracy requirement can be calculated by Eq. (31) in accordance with Eq. (8).

$$
d_{C H_{i} j}=\frac{\delta_{\max }}{\left|\max \left\{n_{C H_{i}}^{j} \cdot z\right\}\right|},
$$

where $n_{C H_{i}}^{j} . z$ is the set of $z$ components of unit normal vectors of the intersected facets of the $j$ th slicing plane and $\mathrm{CH}_{i}$ and $\delta_{\max }$ is the cusp height threshold. The actual layer thickness, $d_{C H_{i},}$, must meet the limit of actual allowable layer thickness, which is written as

$$
d_{C H_{i}, j}= \begin{cases}d_{\max }, & d_{C H_{i} j} \geqslant d_{\max }, \\ d_{\min }, & d_{C H_{i} j} \leqslant d_{\min } .\end{cases}
$$

If the heights of several circular holes overlap one another along the build direction (shown in Fig. 6), then the actual layer thickness of the overlapping area is the smallest value among all layer thicknesses of the relative circular holes and is expressed as

$$
d_{C H_{i}, j}^{\prime}=\min \left\{d_{C H_{i}, j}\right\},
$$

where $d_{C H_{i}, j}^{\prime}$ is the $j$ th layer thickness of the overlapping area.

If a layer of the circular hole crosses the lowest or highest height of the overlapping area, then the excess part needs to be deleted to maintain the integrity of the circular hole feature, and the corresponding layer thickness needs to be recalculated. As shown in Fig. 6, part of the $j$ th layer of $\mathrm{CH}_{1}$ is located in the overlapping area of $\mathrm{CH}_{1}$ and $\mathrm{CH}_{2}$, i.e., $z_{1, j+1}>z_{C H_{2} \text {,min }}$. Hence, the layer thickness $d_{C H_{1}, j}$ of the $j$ th layer needs to be recalculated to make $z_{1, j+1}=z_{\mathrm{CH}_{2} \text {, min }}$. If the last layer thickness of a circular hole is less than the minimum layer thickness (e.g., $\left.d_{C H_{2}, n}<d_{\text {min }}\right)$, then the last several layers need to be recalculated because the last layer cannot be manufactured correctly. The remaining part of the part model is manufactured using the maximum layer thickness $d_{\max }$ to decrease the entire build time. 


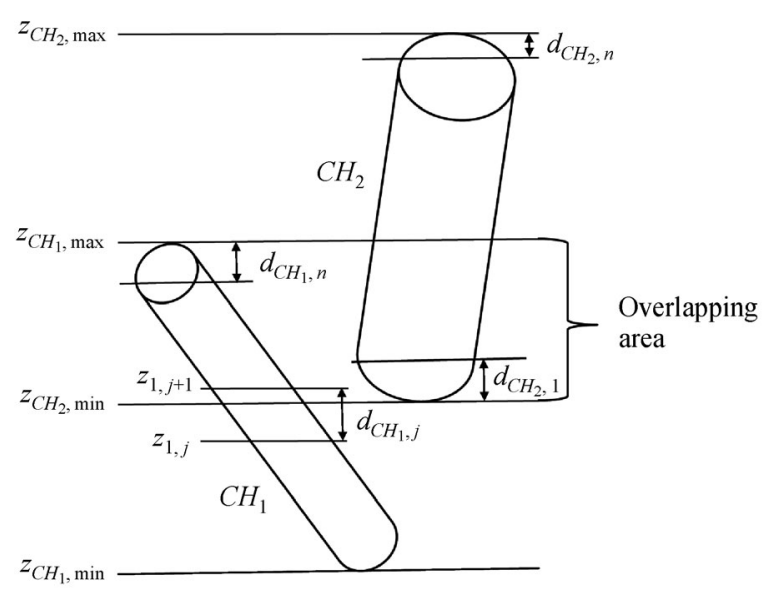

Fig. 6 Overlapping area of two non-planar circular holes along the build direction.

\section{Implementation and results}

The proposed approach is implemented with two mechani- cal models. Figure 7 shows trestle and gearbox models with several circular holes whose axis directions are not parallel to each other. The corresponding surface accuracy requirements of the trestle and gearbox models are also shown in Fig. 7. The size of the trestle model is $20.00 \mathrm{~mm}$ $\times 20.00 \mathrm{~mm} \times 35.00 \mathrm{~mm}$ for $X \times Y \times Z$, and the number of triangular facets is 6890 . The size of the gearbox model is $31.20 \mathrm{~mm} \times 27.60 \mathrm{~mm} \times 12.53 \mathrm{~mm}$ for $X \times Y \times Z$, and the number of triangular facets is 5896 .

The corresponding STL models of the trestle and gearbox with circular closed line loops and the triangular facet sets of the circular holes are shown in Fig. 8. Figures $8(\mathrm{a})$ and $8(\mathrm{c})$ indicate that the circular closed line loops of the trestle and gearbox casing may be located on the outer surface of a cylinder, the inner surface of circular holes, and the transition fillet. Therefore, correct circular closed line loops must be selected to obtain the corresponding triangular facet sets of the circular holes, which are shown in Figs. 8(b) and 8(d). Notably, no accuracy requirement exists for the bolt connection holes of the gearbox; hence, it is not included in the determination of the part build orientation.

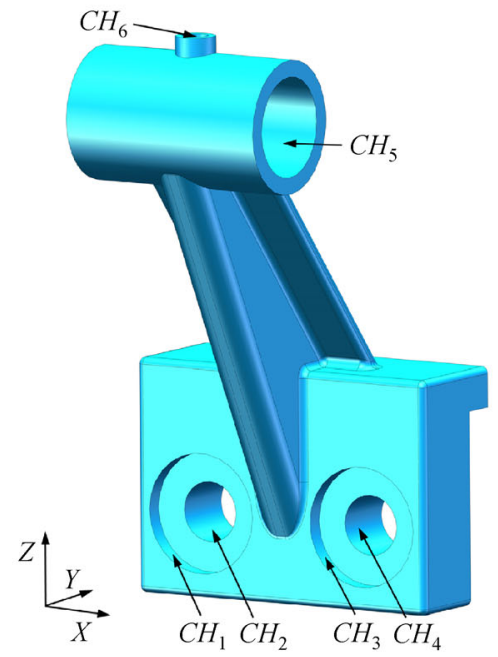

(a)

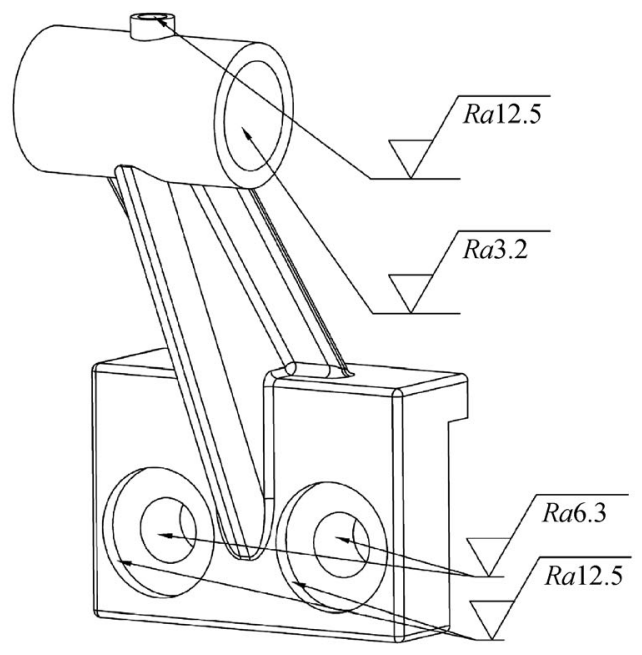

(b)

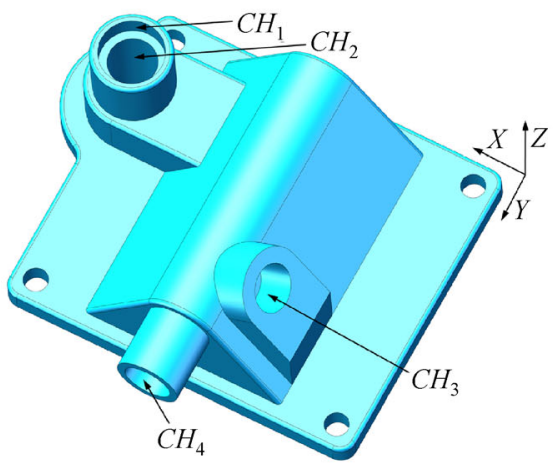

(c)

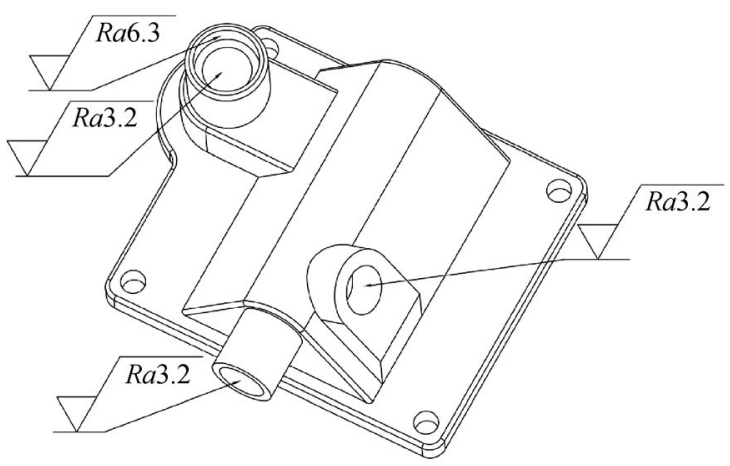

(d)

Fig. 7 (a) Original trestle manifold model with spatial non-planar hole set; (b) trestle surface accuracy requirements on six holes; (c) original gearbox manifold model; (d) gearbox surface accuracy requirements on four holes. Ra: Surface roughness. 


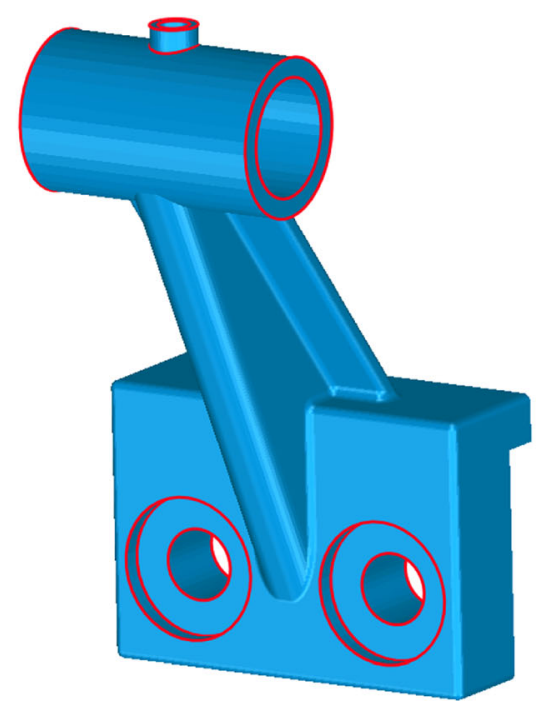

(a)

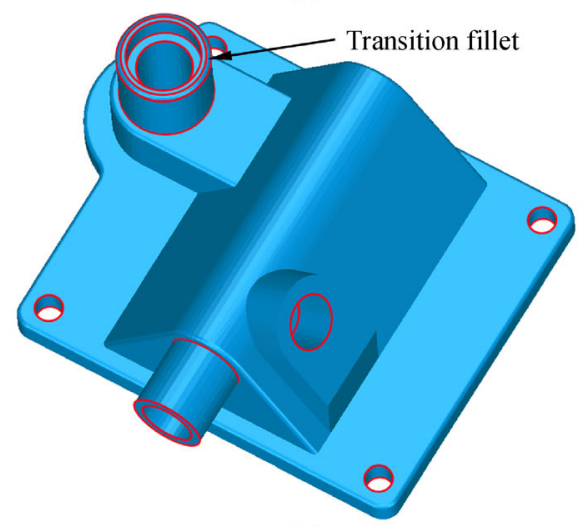

(c)

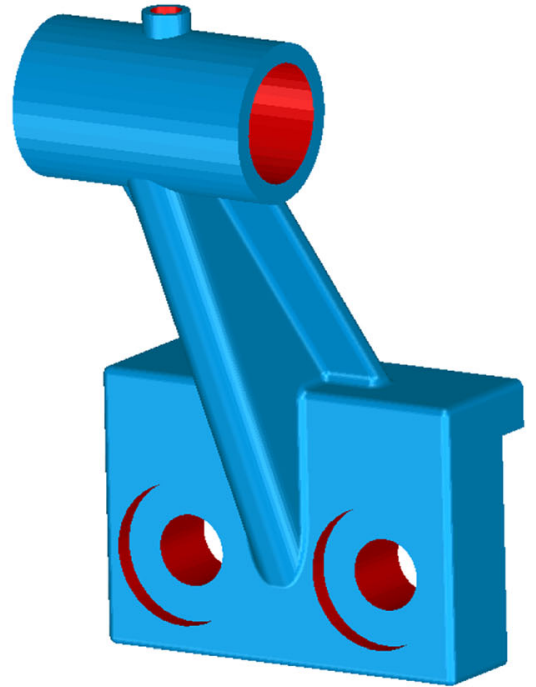

(b)

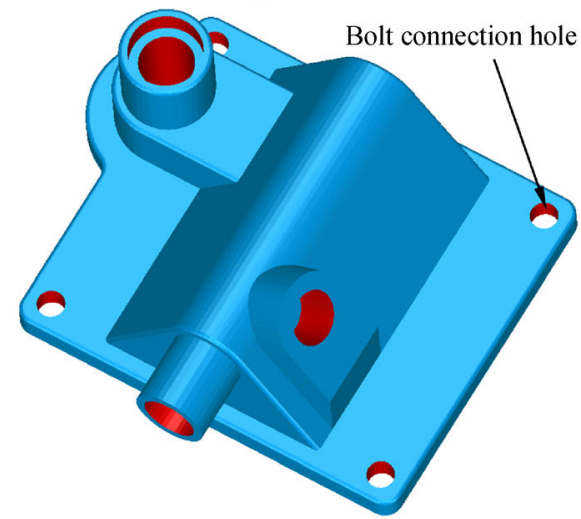

(d)

Fig. 8 (a) Circular closed line loops of the trestle; (b) triangular facet sets of the circular holes of the trestle; (c) circular closed line loops of the gearbox; (d) triangular facet sets of the circular holes of the gearbox.

The circular holes $\mathrm{CH}_{1}, \mathrm{CH}_{2}, \mathrm{CH}_{3}$, and $\mathrm{CH}_{4}$ of the trestle have the same axis direction, but the axis directions of $\mathrm{CH}_{5}$ and $\mathrm{CH}_{6}$ of the trestle are perpendicular to it. The same situation applies to the gearbox. The information of the six circular holes of the trestle is shown in Table 2, and the information of the four circular holes of the gearbox is shown in Table 3. Selecting a perfect part build orientation for the trestle and gearbox by direct assignment is difficult. The minimum and maximum allowable layer thicknesses are 0.1 and $0.3 \mathrm{~mm}$, respectively, and the cusp height threshold is set to $0.1 \mathrm{~mm}$.

The surface accuracy requirements of the circular holes of the trestle indicate that $\mathrm{CH}_{1}, \mathrm{CH}_{3}$, and $\mathrm{CH}_{6}$ have the same weight. $\mathrm{CH}_{2}$ and $\mathrm{CH}_{4}$ also have a same weight. The trestle has only three surface accuracy requirements. $\mathrm{CH}_{5}$ has the highest surface accuracy requirement among $\mathrm{CH}_{1}$, $\mathrm{CH}_{2}$, and $\mathrm{CH}_{5} . \mathrm{CH}_{1}$ has the lowest surface accuracy requirement among $\mathrm{CH}_{1}, \mathrm{CH}_{2}$, and $\mathrm{CH}_{5}$. Meanwhile, the surface accuracy requirement of $\mathrm{CH}_{2}$ is between those of $\mathrm{CH}_{5}$ and $\mathrm{CH}_{1}$. For the judgment to have sufficient

Table 2 Circular hole information of the trestle

\begin{tabular}{|c|c|c|c|c|c|}
\hline Circular hole & Axis direction & Diameter/mm & Depth/mm & Facet number & Weight \\
\hline $\mathrm{CH}_{1}$ & $(0.0000,1.0000,0.0000)$ & 7.2 & 1.00 & 76 & 0.0591 \\
\hline $\mathrm{CH}_{2}$ & $(0.0000,1.0000,0.0000)$ & 3.6 & 4.00 & 72 & 0.1523 \\
\hline $\mathrm{CH}_{3}$ & $(0.0000,1.0000,0.0000)$ & 7.2 & 1.00 & 76 & 0.0591 \\
\hline $\mathrm{CH}_{4}$ & $(0.0000,1.0000,0.0000)$ & 3.6 & 4.00 & 72 & 0.1523 \\
\hline $\mathrm{CH}_{5}$ & $(1.0000,0.0000,0.0000)$ & 6.0 & 13.00 & 160 & 0.5181 \\
\hline $\mathrm{CH}_{6}$ & $(0.0000,0.0000,1.0000)$ & 1.6 & 2.11 & 122 & 0.0591 \\
\hline
\end{tabular}


Table 3 Circular hole information of the gearbox

\begin{tabular}{|c|c|c|c|c|c|}
\hline Circular hole & Axis direction & Diameter/mm & Depth/mm & Facet number & Weight \\
\hline $\mathrm{CH}_{1}$ & $(0.0000,0.0000,1.0000)$ & 4.8 & 1.8 & 72 & 0.0631 \\
\hline $\mathrm{CH}_{2}$ & $(0.0000,0.0000,1.0000)$ & 3.6 & 9.6 & 72 & 0.3123 \\
\hline $\mathrm{CH}_{3}$ & $(-0.8223,0.0000,0.5691)$ & 3.6 & 3.0 & 72 & 0.3123 \\
\hline $\mathrm{CH}_{4}$ & $(0.0000,1.0000,0.0000)$ & 3.6 & 9.6 & 72 & 0.3123 \\
\hline
\end{tabular}

discrimination, the importance of $\mathrm{CH}_{2}$ relative to $\mathrm{CH}_{1}$ is regarded as moderate, and the corresponding TFN is $M_{3}$. The importance of $\mathrm{CH}_{5}$ relative to $\mathrm{CH}_{2}$ is considered essential, and the corresponding TFN is $M_{5}$. The reason for considering that the importance of $\mathrm{CH}_{5}$ relative to $\mathrm{CH}_{2}$ is $M_{5}$ and the reason for considering that the importance of $\mathrm{CH}_{2}$ relative to $\mathrm{CH}_{1}$ is $\mathrm{M}_{3}$ are as follows: As the accuracy requirements increase, the machining becomes increasingly difficult. Therefore, the importance of $\mathrm{CH}_{5}$ relative to $\mathrm{CH}_{1}$ is regarded as high for the same reason, and the corresponding TFN is $M_{7}$. Similarly, a pairwise comparison of the circular holes of the gearbox can be performed. The TFN judgment matrices of the trestle and gearbox are obtained as follows:

$$
\begin{aligned}
& \boldsymbol{Q}_{1}=\left(\begin{array}{cccccc}
(1,1,1) & (1 / 4,1 / 3,1 / 2) & (1,1,1) & (1 / 4,1 / 3,1 / 2) & (1 / 8,1 / 7,1 / 6) & (1,1,1) \\
(2,3,4) & (1,1,1) & (2,3,4) & (1,1,1) & (1 / 6,1 / 5,1 / 4) & (2,3,4) \\
(1,1,1) & (1 / 4,1 / 3,1 / 2) & (1,1,1) & (1 / 4,1 / 3,1 / 2) & (1 / 8,1 / 7,1 / 6) & (1,1,1) \\
(2,3,4) & (1,1,1) & (2,3,4) & (1,1,1) & (1 / 6,1 / 5,1 / 4) & (2,3,4) \\
(6,7,8) & (4,5,6) & (6,7,8) & (4,5,6) & (1,1,1) & (6,7,8) \\
(1,1,1) & (1 / 4,1 / 3,1 / 2) & (1,1,1) & (1 / 4,1 / 3,1 / 2) & (1 / 8,1 / 7,1 / 6) & (1,1,1)
\end{array}\right), \\
& \boldsymbol{Q}_{2}=\left(\begin{array}{cccc}
(1,1,1) & (1 / 6,1 / 5,1 / 4) & (1 / 6,1 / 5,1 / 4) & (1 / 6,1 / 5,1 / 4) \\
(4,5,6) & (1,1,1) & (1,1,1) & (1,1,1) \\
(4,5,6) & (1,1,1) & (1,1,1) & (1,1,1) \\
(4,5,6) & (1,1,1) & (1,1,1) & (1,1,1)
\end{array}\right) .
\end{aligned}
$$

The corresponding reciprocal non-fuzzy judgment matrices are as follows:

$$
\boldsymbol{R}_{1}=\left(\begin{array}{llllll}
1.0000 & 0.3436 & 1.0000 & 0.3436 & 0.1436 & 1.0000 \\
2.9104 & 1.0000 & 2.9104 & 1.0000 & 0.2021 & 2.9104 \\
1.0000 & 0.3436 & 1.0000 & 0.3436 & 0.1436 & 1.0000 \\
2.9104 & 1.0000 & 2.9104 & 1.0000 & 0.2021 & 2.9104 \\
6.9649 & 4.9483 & 6.9649 & 4.9483 & 1.0000 & 6.9649 \\
1.0000 & 0.3436 & 1.0000 & 0.3436 & 0.1436 & 1.0000
\end{array}\right)
$$

$$
\boldsymbol{R}_{2}=\left(\begin{array}{llll}
1.0000 & 0.2021 & 0.2021 & 0.2021 \\
4.9483 & 1.0000 & 1.0000 & 1.0000 \\
4.9483 & 1.0000 & 1.0000 & 1.0000 \\
4.9483 & 1.0000 & 1.0000 & 1.0000
\end{array}\right)
$$

The random consistency ratio of the trestle is $C R_{1}=$ $0.0142<0.1000$. The random consistency ratio of the gearbox is $C R_{2}=0.0014<0.1000$. Both $\boldsymbol{R}_{1}$ and $\boldsymbol{R}_{2}$ pass the AHP consistency test. The weight vectors of the trestle and gearbox are as follows:

$$
\begin{aligned}
& \boldsymbol{W}_{1}=\left(\begin{array}{llll}
0.0591 & 0.1523 & 0.0591 & 0.1523 \\
0.5181 & 0.0591
\end{array}\right)^{\mathrm{T}} \\
& \boldsymbol{W}_{2}=\left(\begin{array}{llll}
0.0631 & 0.3123 & 0.3123 & 0.3123
\end{array}\right)^{\mathrm{T}} .
\end{aligned}
$$


The optimal part build orientation of the trestle and gearbox can be obtained through the method proposed in Section 3. The layer thickness for determining the part build orientation is $0.1 \mathrm{~mm}$. The WVE objective function landscapes of Eq. (23) of the trestle and gearbox are shown in Figs. 9(a) and 9(b), respectively. The optimal orientation of the trestle is $\left(\forall \theta_{x}, 90^{\circ}\right) . \forall \theta_{x}$ means that for all $\theta_{x} \in\left[0^{\circ}\right.$, $180^{\circ}$ ], the WVE objective function values of the optimal orientation and original orientation of the trestle are 4.6310 and $9.9205 \mathrm{~mm}^{3}$, respectively. The optimal orientation of the gearbox is $\left(90^{\circ}, 0^{\circ}\right)$ or $\left(90^{\circ}, 180^{\circ}\right)$. Another evaluation standard is considered in this case to select a good solution. The final build time of each solution is regarded as the evaluation standard. Therefore, the solution $\left(90^{\circ}, 0^{\circ}\right)$ is taken as the optimal orientation because it has less build time than the solution $\left(90^{\circ}, 180^{\circ}\right)$. The solution $\left(90^{\circ}, 0^{\circ}\right)$ has less build time than the solution $\left(90^{\circ}, 180^{\circ}\right)$ because the solution $\left(90^{\circ}, 0^{\circ}\right)$ has fewer supports. The WVE objective function values of the optimal orientation and original orientation of the gearbox are 6.1266 and 14.9043 $\mathrm{mm}^{3}$, respectively.

The uniform and adaptive slicing results of the original and optimal orientations of the trestle are shown in Fig. 10. The layer thickness of uniform slicing at original and optimal orientations is $0.1 \mathrm{~mm}$. The number of slicing layers of uniform slicing at the original orientation is 350 , and the numbers of slicing layers of uniform and adaptive slicing at the optimal orientation are 200 and 120, respectively.

A comparison of the cusp heights of the three slicing results for the trestle is shown in Fig. 11(a). The maximum cusp height of each slicing layer with uniform slicing at the original and optimal orientation meets the accuracy threshold requirement, but several maximum cusp heights of the slicing layers with adaptive slicing at the optimal orientation are larger than the threshold. This result is obtained because the cusp heights of all the triangular facets of each slicing layer are calculated with the inclusion of the cusp heights of the triangular facets of the noncircular hole part. Figure 11(b) shows the maximum cusp heights of all slicing layers of all circular holes for the trestle with adaptive slicing at the optimal orientation. The maximum cusp heights of all circular holes meet the accuracy threshold requirement. Therefore, the number of slicing layers of adaptive slicing is reduced by $40 \%$ compared with that of uniform slicing at the optimal orientation while maintaining the forming surface accuracy of all circular holes of the trestle. As shown in Fig. 11(b), the cusp heights of $\mathrm{CH}_{5}$ with the highest surface accuracy requirement are all zero, but the value is not zero for uniform slicing at the original orientation, as indicated in Fig. 11(a). Therefore, the surface accuracy of $\mathrm{CH}_{5}$ is optimized.

The uniform and adaptive slicing results at the original and optimal orientation of the gearbox are shown in Fig. 12. The layer thickness of uniform slicing at the original and optimal orientation is $0.1 \mathrm{~mm}$. The number of slicing layers of uniform slicing at the original orientation is 125 , and the numbers of slicing layers of uniform and adaptive slicing at the optimal orientation are 276 and 117 , respectively. A comparison of the cusp heights of the three slicing results for the gearbox is shown in Fig. 13(a). Figure 13(b) shows the maximum cusp heights of all slicing layers of all circular holes for the gearbox with adaptive slicing at the optimal orientation. The maximum cusp heights of all circular holes meet the accuracy threshold requirement. The number of slicing layers of adaptive slicing is reduced by $57.61 \%$ compared with that of uniform slicing at the optimal orientation while maintaining the forming surface accuracy of all circular

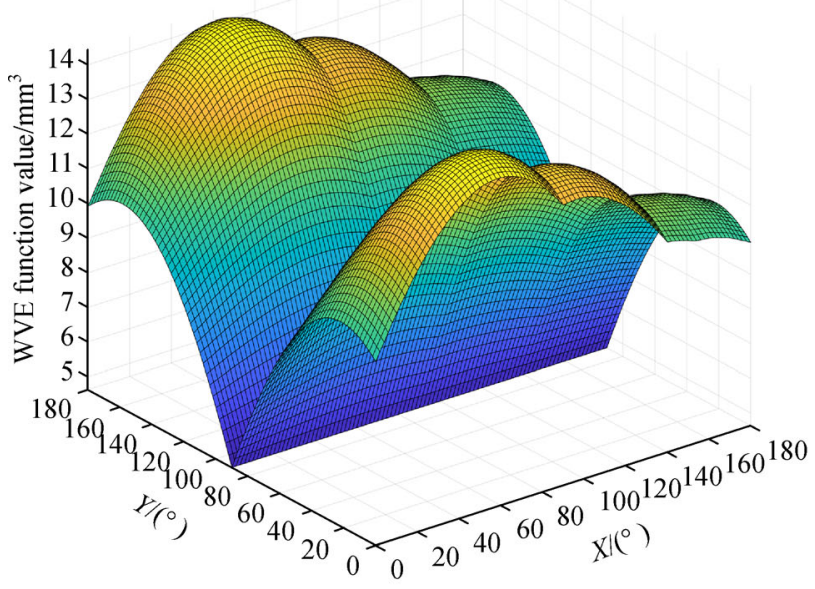

(a)

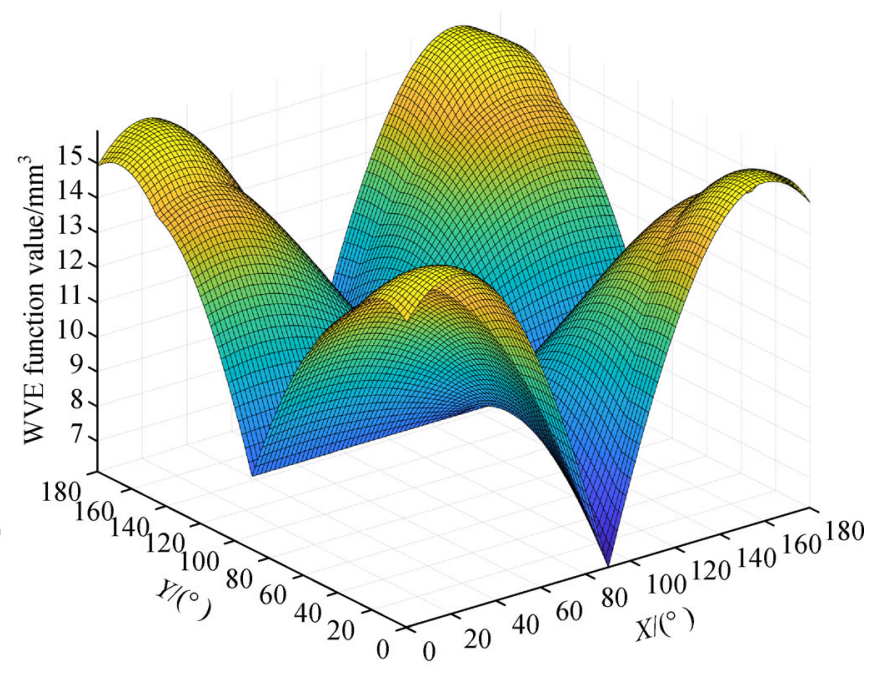

(b)

Fig. 9 WVE objective function landscapes: (a) Trestle and (b) gearbox. 


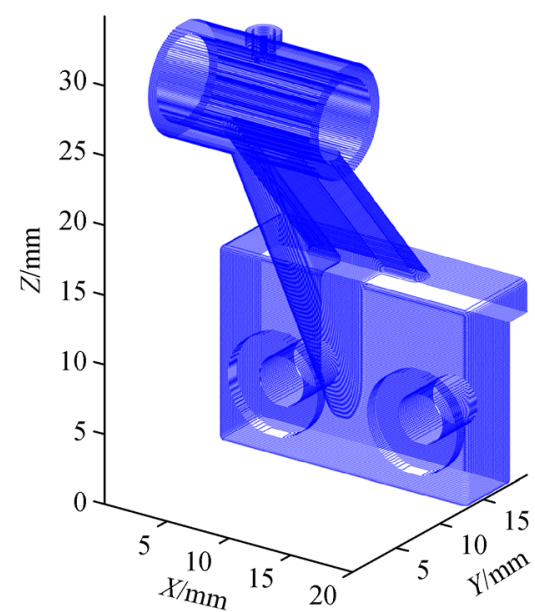

(a)

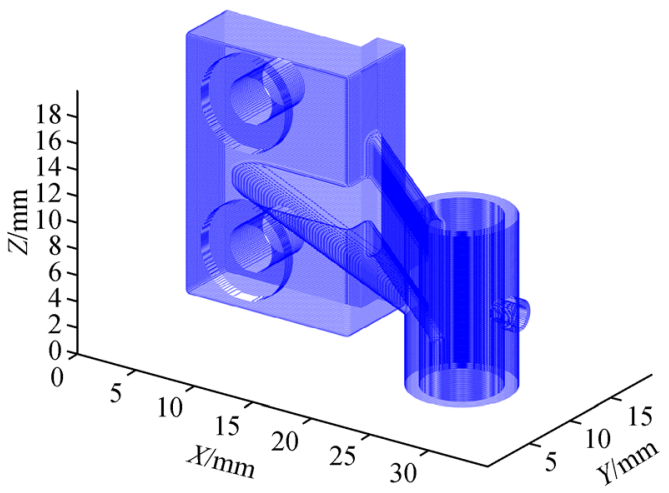

(c)

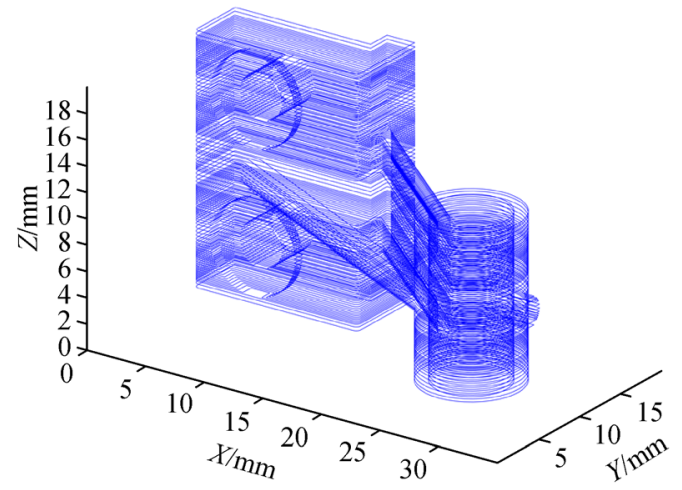

(e)

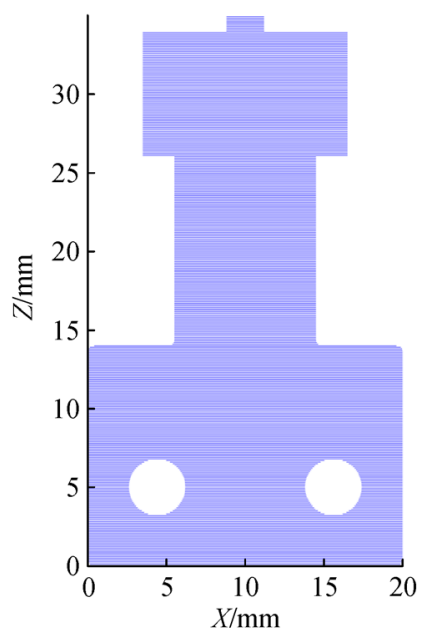

(b)

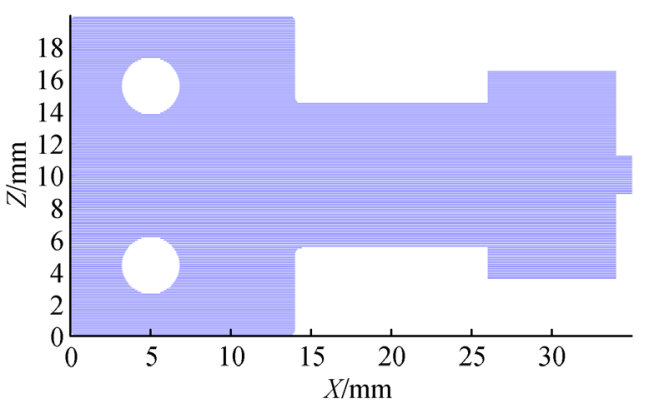

(d)

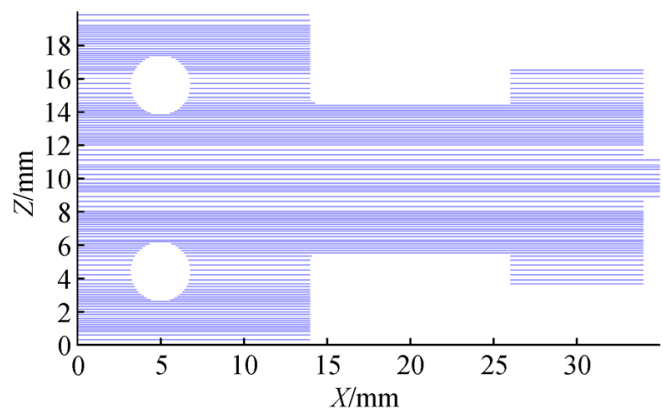

(f)

Fig. 10 Slicing results at the original and optimal build orientation of the trestle. (a) Uniform slicing at the original orientation; (b) uniform slicing at the original orientation on $X Z$ plane; (c) uniform slicing at the optimal orientation; (d) uniform slicing at the optimal orientation on $X Z$ plane; (e) adaptive slicing at the optimal orientation; (f) adaptive slicing at the optimal orientation on $X Z$ plane.

holes of the gearbox. As shown in Fig. 13(b), the cusp heights of $\mathrm{CH}_{4}$ with the highest surface accuracy requirement are all zero. The surface accuracy of $\mathrm{CH}_{4}$ is optimized. The proposed approach is effectively verified by the trestle and gearbox models.

The visualized virtual printing using digital twins of the corresponding slicing results for the trestle and gearbox are shown in Figs. 14 and 15, respectively. The aim is to further demonstrate the effectiveness of the proposed approach. In the figures, the yellow and red parts are the fill part, and the green and deep-green parts are the supports.

The fabricated models obtained after stripping the external supports of the corresponding slicing results for Figs. 14 and 15 are shown in Figs. 16 and 17, respectively. Figures 16(a) and 17(a) are the fabricated models of the trestle and gearbox at the original orientation with uniform 


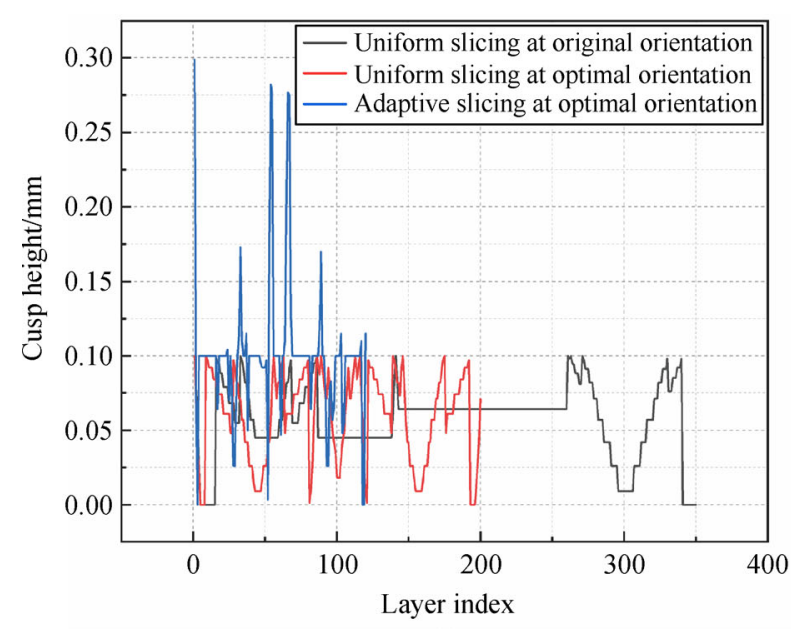

(a)

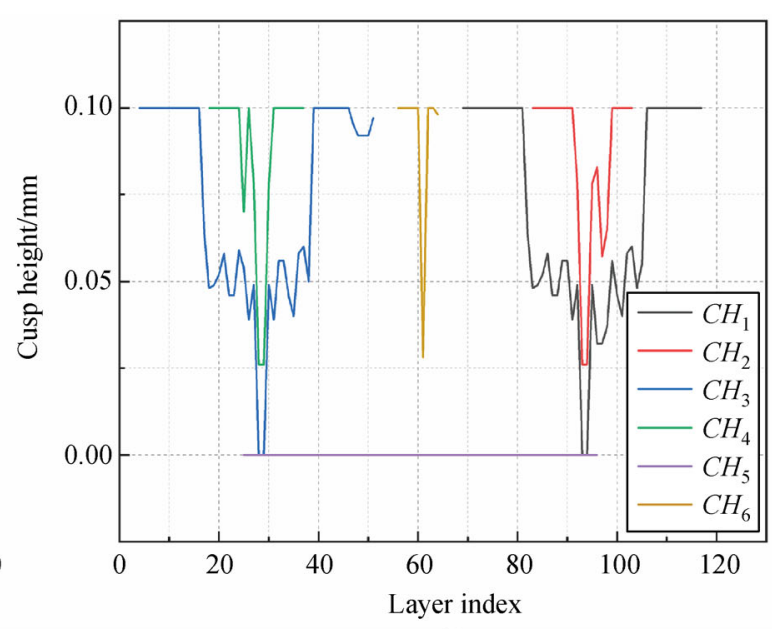

(b)

Fig. 11 Cusp heights of the trestle. (a) Three slicing results; (b) six circular holes with adaptive slicing at the optimal orientation.

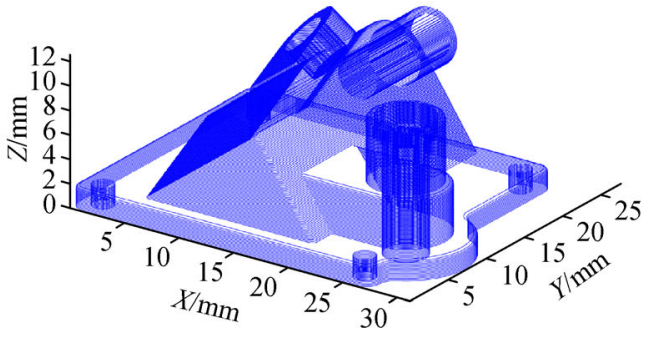

(a)

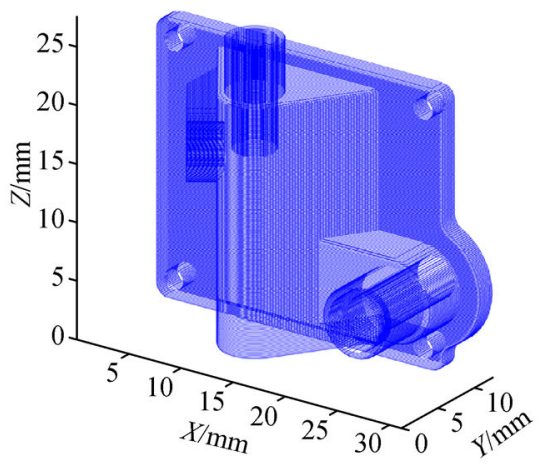

(c)

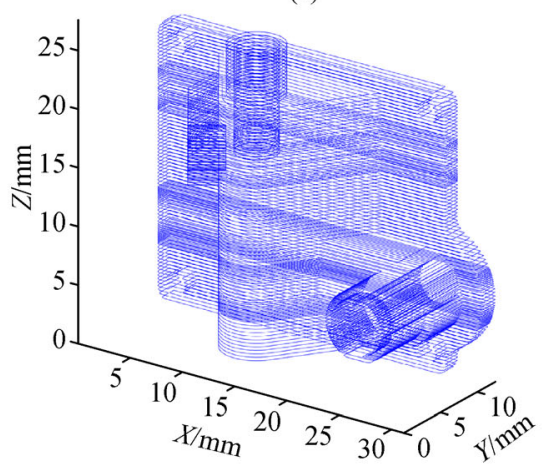

(e)

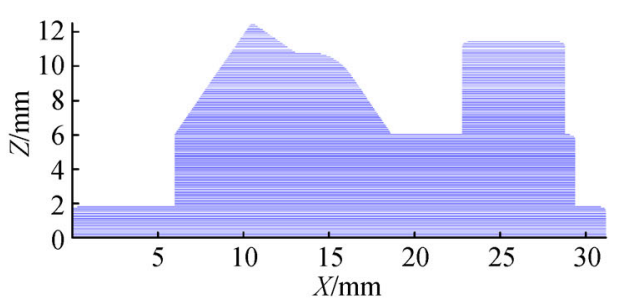

(b)

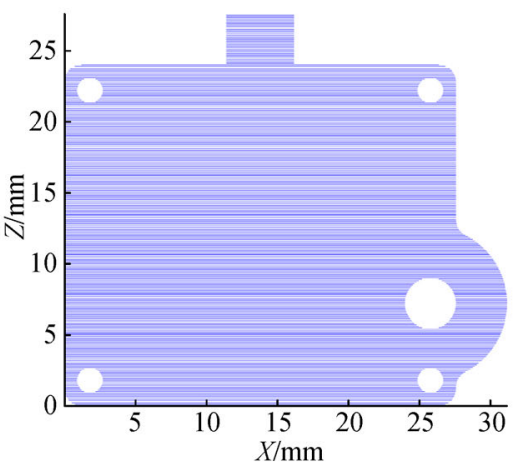

(d)

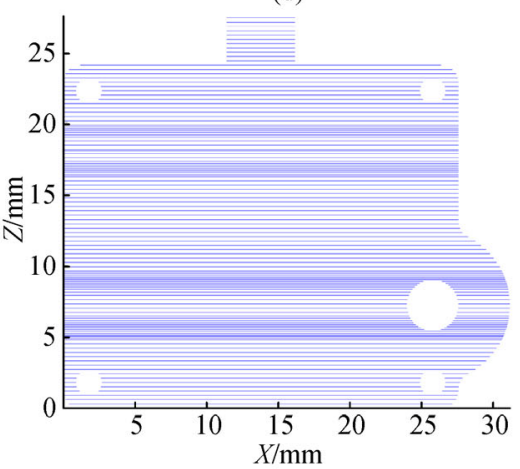

(f)

Fig. 12 Slicing results at the original and optimal build orientation of the gearbox. (a) Uniform slicing at the original orientation; (b) uniform slicing at the original orientation on $X Z$ plane; (c) uniform slicing at the optimal orientation; (d) uniform slicing at the optimal orientation on $X Z$ plane; (e) adaptive slicing at the optimal orientation; (f) adaptive slicing at the optimal orientation on $X Z$ plane. 


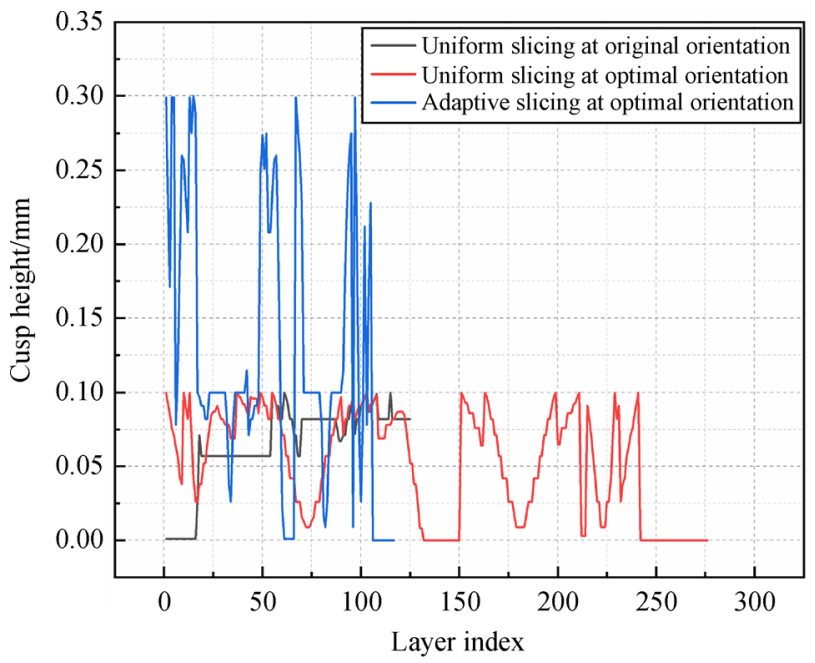

(a)

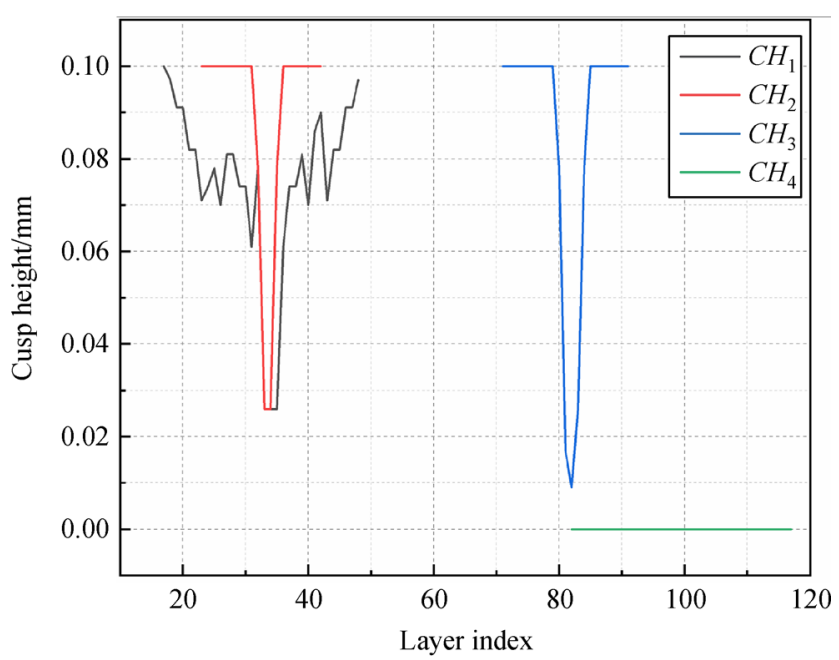

(b)

Fig. 13 Cusp heights of the gearbox. (a) Three slicing results; (b) four circular holes with adaptive slicing at the optimal orientation.

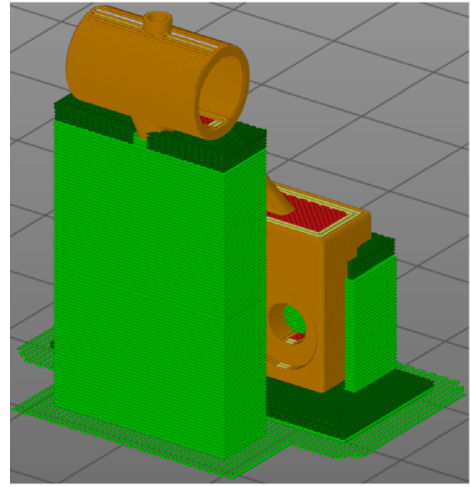

(a)

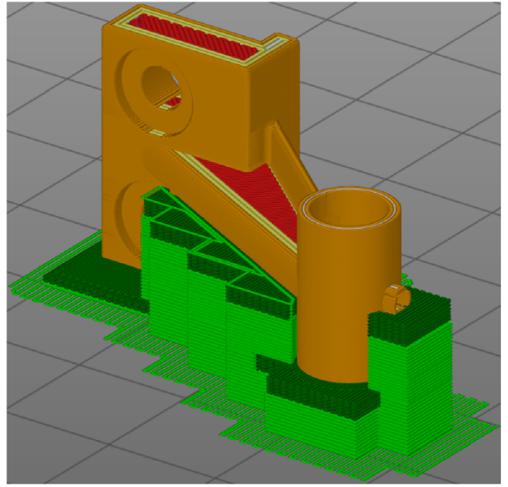

(b)

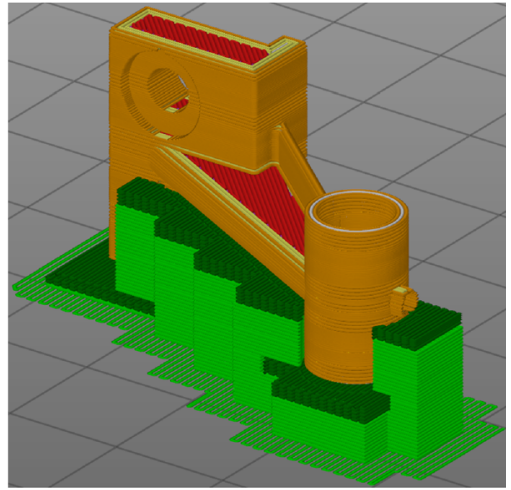

(c)

Fig. 14 Visualized virtual printing using digital twins for the trestle. (a) Uniform slicing at the original orientation; (b) uniform slicing at the optimal orientation; (c) adaptive slicing at the optimal orientation.

slicing, respectively. Figures $16(\mathrm{~b})$ and $16(\mathrm{c})$ are the fabricated models of the trestle at the optimal orientation with uniform and adaptive slicing, respectively. The build time of Fig. 16(c) is less than that in Fig. 16(b). Figures $17(\mathrm{~b})$ and $17(\mathrm{c})$ are the fabricated models of the gearbox for the solution $\left(90^{\circ}, 0^{\circ}\right)$ and solution $\left(90^{\circ}, 180^{\circ}\right)$ with uniform slicing, respectively. Figure 17(b) is taken as the final optimal orientation because it has less build time. Figure 17(d) is the fabricated model of the gearbox at the optimal orientation with adaptive slicing. The build time of Fig. 17(d) is less than that in Fig. 17(b).

\section{Conclusions}

This study proposes a surface accuracy optimization approach based on TFN to optimize the surface accuracy of the circular holes of mechanical parts fabricated by AM. The circular holes of a part's STL manifold model are determined using the dihedral angle method and normal tensor voting theory. Then, the optimal part build orientation is acquired using GA with fuzzy weights for the circular holes to minimize the WVE of a part. The corresponding weights are obtained based on the surface accuracy requirements of different circular holes by using the AHP method based on TFN. Afterward, the entire build time is reduced by adopting an improved adaptive slicing algorithm while maintaining the forming surface accuracy of the circular holes. The implementation results of the trestle and gearbox demonstrate the effectiveness of the proposed approach.

In the future, the establishment of TFN judgment matrices for a part with numerous non-planar circular holes can be optimized by analyzing and classifying all circular holes based on the surface accuracy requirements. Moreover, the multi-objective optimization algorithm, which includes other objectives (e.g., surface roughness and support structure), can be utilized to determine the 


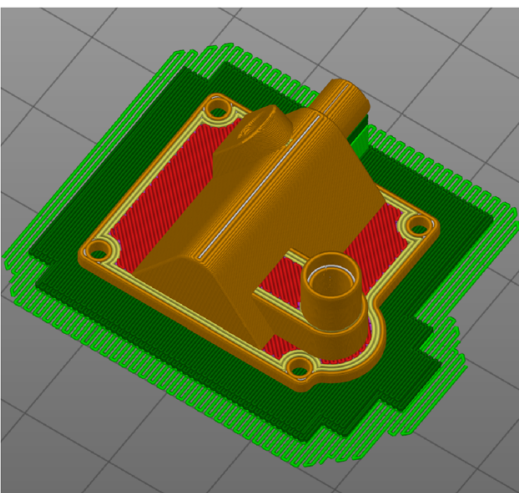

(a)

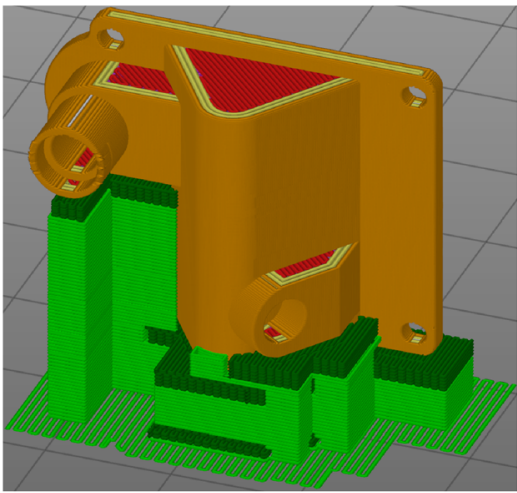

(c)

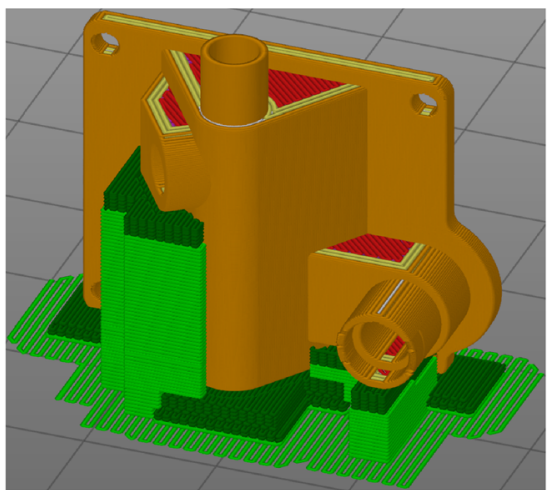

(b)

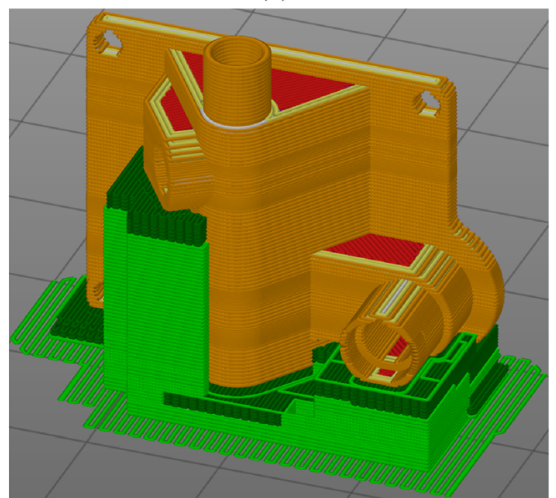

(d)

Fig. 15 Visualized virtual printing using digital twins for the gearbox. (a) Uniform slicing at the original orientation; (b) uniform slicing at the optimal orientation $\left(90^{\circ}, 0^{\circ}\right)$; (c) uniform slicing at the optimal orientation $\left(90^{\circ}, 180^{\circ}\right)$; (d) adaptive slicing at the optimal orientation.

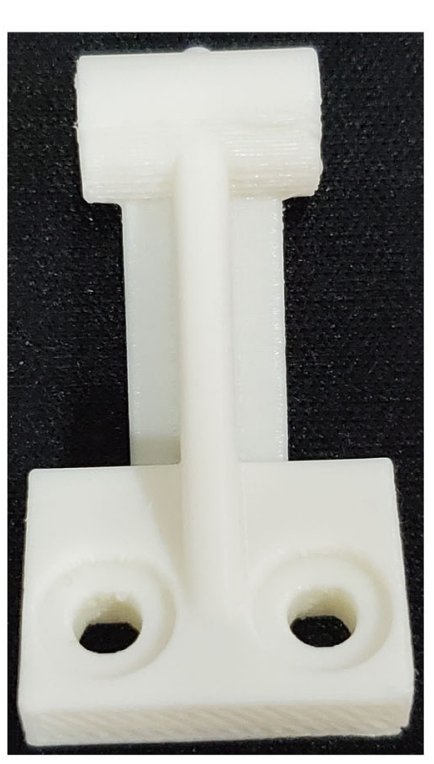

(a)

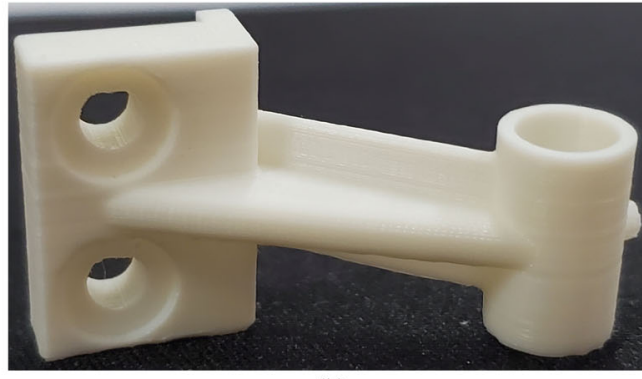

(b)

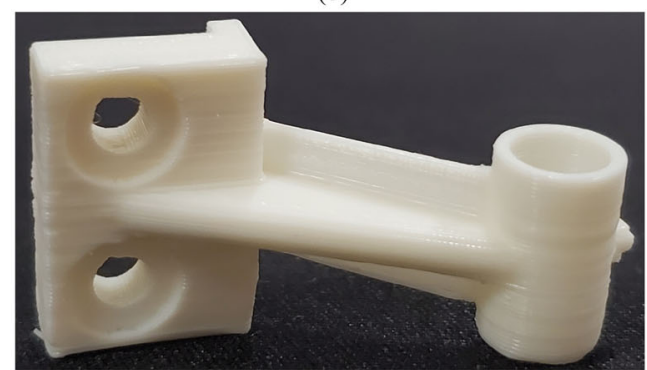

(c)

Fig. 16 Fabricated trestle models after stripping the supports for (a) Fig. 14(a), (b) Fig. 14(b), and (c) Fig. 14(c). 


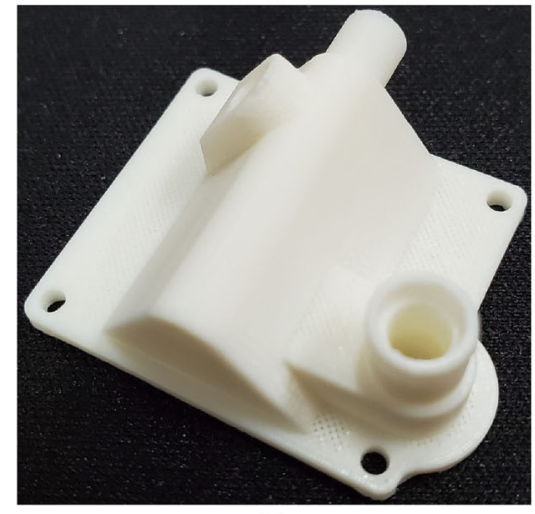

(a)

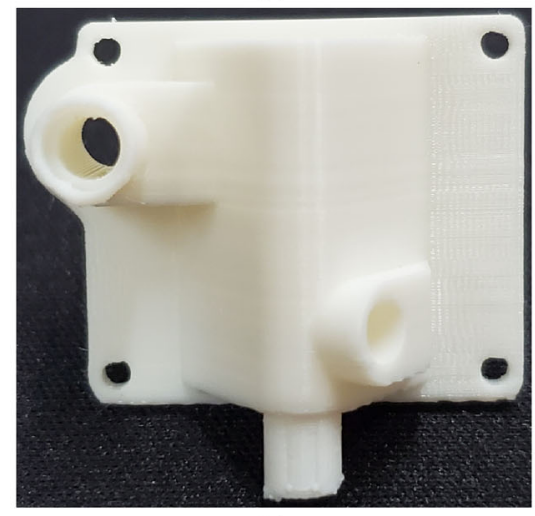

(c)

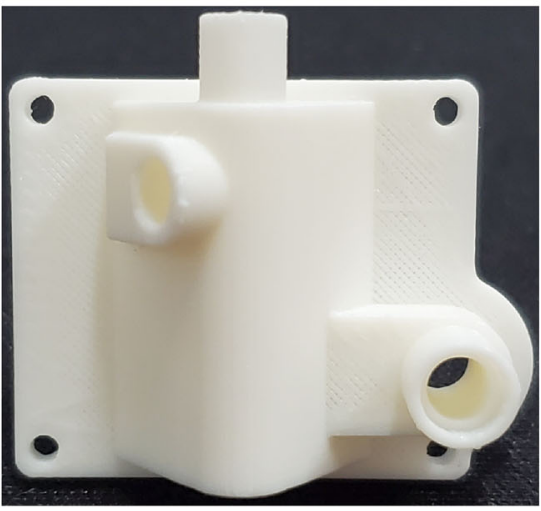

(b)

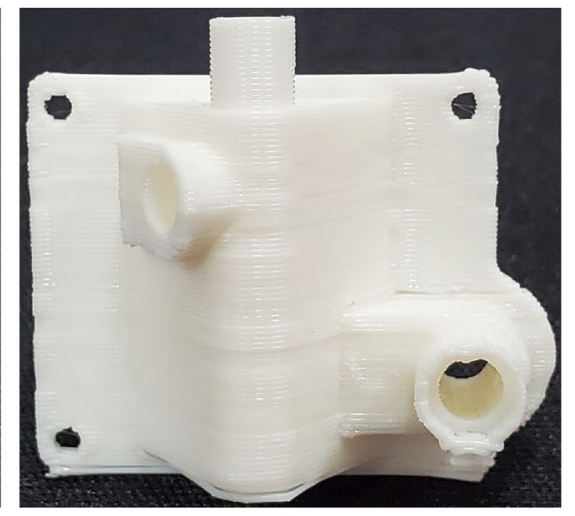

(d)

Fig. 17 Fabricated gearbox castings after stripping the external supports for (a) Fig. 15(a), (b) Fig. 15(b), (c) Fig. 15(c), and (d) Fig. 15(d).

optimal part build orientation for improving the surface accuracy and surface quality of the circular holes of a part comprehensively.

Acknowledgements This work was supported by the National Natural Science Foundation of China (Grant Nos. 51775494, 51821093, and 51935009), the National Key R\&D Program of China (Grant No. 2018YFB1700701), the Science and Technology Project of Zhejiang Province, China (Grant No. 2019C01141), and the Zhejiang Provincial Basic Public Welfare Research Project, China (Grant Nos. LGG18E050007 and LGG21E050020).

Open Access This article is licensed under a Creative Commons Attribution 4.0 International License, which permits use, sharing, adaptation, distribution, and reproduction in any medium or format as long as you give appropriate credit to the original author(s) and the source, provide a link to the Creative Commons license, and indicate if changes were made.

The images or other third-party material in this article are included in the article's Creative Commons license, unless indicated otherwise in a credit line to the material. If a material is not included in the article's Creative Commons license and your intended use is not permitted by statutory regulation or exceeds the permitted use, you will need to obtain permission directly from the copyright holder.

To view a copy of this license, visit http://creativecommons.org/licenses/ by/4.0\%.

\section{References}

1. Cao Z L, Liu Z D, Ling J J, et al. Deep-type hole machining by inner jetted aerosol dielectric ablation. International Journal of Advanced Manufacturing Technology, 2015, 78(9-12): 1989-1998

2. Dudak N, Taskarina A, Kasenov A, et al. Hole machining based on using an incisive built-up reamer. International Journal of Precision Engineering and Manufacturing, 2017, 18(10): 1425-1432

3. Tie Y, Zhou X H, Li C, et al. Effect of hole machining method on the behavior of CFRP laminates under low-velocity impacts. Mechanics of Composite Materials, 2018, 54(3): 369-378

4. Hocheng H, Tsao C C. Effects of special drill bits on drillinginduced delamination of composite materials. International Journal of Machine Tools and Manufacture, 2006, 46(12-13): 1403-1416

5. Steuben J C, Iliopoulos A P, Michopoulos J G. Implicit slicing for functionally tailored additive manufacturing. Computer-Aided Design, 2016, 77: 107-119

6. Murr L E. Frontiers of 3D printing/additive manufacturing: From human organs to aircraft fabrication. Journal of Materials Science and Technology, 2016, 32(10): 987-995

7. Dolenc A, Makela I. Slicing procedures for layered manufacturing techniques. Computer-Aided Design, 1994, 26(2): 119-126

8. Rattanawong W, Masood S H, Iovenitti P. A volumetric approach to part-build orientations in rapid prototyping. Journal of Materials Processing Technology, 2001, 119(1-3): 348-353

9. Byun H S, Lee K H. Determination of the optimal part orientation in layered manufacturing using a genetic algorithm. International Journal of Production Research, 2005, 43(13): 2709-2724

10. Zhao J B. Determination of optimal orientation based on satisfactory degree theory for RPT. In: Proceedings of the Ninth International 
Conference on Computer Aided Design and Computer Graphics. Hongkong: IEEE, 2005, 225-230

11. Luo N, Wang Q. Fast slicing orientation determining and optimizing algorithm for least volumetric error in rapid prototyping. International Journal of Advanced Manufacturing Technology, 2016, 5-8(83): 1297-1313

12. Ezair B, Massarwi F, Elber G. Orientation analysis of 3D objects toward minimal support volume in 3D-printing. Computers \& Graphics, 2015, 51: 117-124

13. Pereira S, Vaz A I F, Vicente L N. On the optimal object orientation in additive manufacturing. International Journal of Advanced Manufacturing Technology, 2018, 98(5-8): 1685-1694

14. Miyanaji H, Orth M, Akbar J M, et al. Process development for green part printing using binder jetting additive manufacturing. Frontiers of Mechanical Engineering, 2018, 13(4): 504-512

15. Shan Z D, Guo Z, Du D, et al. Digital high-efficiency print forming method and device for multi-material casting molds. Frontiers of Mechanical Engineering, 2020, 15(2): 328-337

16. Zhang Z, Joshi S. An improved slicing algorithm with efficient contour construction using STL files. International Journal of Advanced Manufacturing Technology, 2015, 80(5-8): 1347-1362

17. Zeng L, Lai M L, Qi D, et al. Efficient slicing procedure based on adaptive layer depth normal image. Computer-Aided Design, 2011, 43(12): 1577-1586

18. Qi D, Zeng L, Yuen M F. Robust slicing procedure based on Surfelgrid. Computer-Aided Design and Applications, 2013, 10(6): 965 981

19. Kulkarni P, Dutta D. An accurate slicing procedure for layered manufacturing. Computer-Aided Design, 1996, 28(9): 683-697

20. Gupta S, Prusty R K, Ray B C. Strength degradation and fractographic analysis of carbon fiber reinforced polymer composite laminates with square/circular hole using scanning electron microscope micrographs. Journal of Applied Polymer Science, 2021, 138(8): 49878

21. Ma G F, Kang R K, Dong Z G, et al. Hole quality in longitudinaltorsional coupled ultrasonic vibration assisted drilling of carbon fiber reinforced plastics. Frontiers of Mechanical Engineering, 2020, 15(4): 538-546

22. Rianmora S, Koomsap P. Recommended slicing positions for adaptive direct slicing by image processing technique. The International Journal of Advanced Manufacturing Technology, 2010, 46(9-12): 1021-1033

23. Hayasi M T, Asiabanpour B. A new adaptive slicing approach for the fully dense freeform fabrication (FDFF) process. Journal of Intelligent Manufacturing, 2013, 24(4): 683-694

24. Butt J, Onimowo D A, Gohrabian M, et al. A desktop 3D printer with dual extruders to produce customised electronic circuitry. Frontiers of Mechanical Engineering, 2018, 13(4): 528-534

25. Ohtake Y, Belyaev A, Seidel H P. Ridge-valley lines on meshes via implicit surface fitting. ACM Transactions on Graphics, 2004, 23(3): 609-612

26. $\mathrm{Kim} \mathrm{S} \mathrm{K,} \mathrm{Kim} \mathrm{C} \mathrm{H.} \mathrm{Finding} \mathrm{ridges} \mathrm{and} \mathrm{valleys} \mathrm{in} \mathrm{a} \mathrm{discrete} \mathrm{surface}$ using a modified MLS approximation. Computer-Aided Design, 2006, 38(2): 173-180

27. Sunil V B, Pande S S. Automatic recognition of features from freeform surface CAD models. Computer-Aided Design, 2008, 40 (4): 502-517

28. Shimizu T, Date H, Kanai S, et al. A new bilateral mesh smoothing by recognizing features. In: Proceedings of the Ninth International Conference on Computer Aided Design and Computer Graphics. Hongkong: IEEE Computer Society Press, 2005, 281-286

29. Kim H S, Choi H K, Lee K H. Feature detection of triangular meshes based on tensor voting theory. Computer-Aided Design, 2009, 41 (1): $47-58$

30. Jiao X M, Bayyana N R. Identification of $C^{1}$ and $C^{2}$ discontinuities for surface meshes in CAD. Computer-Aided Design, 2008, 40(2): 160-175

31. Qu X Z, Stucker B. Circular hole recognition for STL-based toolpath generation. Rapid Prototyping Journal, 2005, 11(3): 132139

32. Yang X N, Zheng J M, Wang D S. A computational approach to joint line detection on triangular meshes. Engineering with Computers, 2014, 30(4): 583-597

33. Tong W H, Tai X C. A variational approach for detecting feature lines on meshes. Journal of Computational Mathematics, 2016, 34(1): 87-112

34. Ghasemi H, Park H S, Rabczuk T. A multi-material level set-based topology optimization of flexoelectric composites. Computer Methods in Applied Mechanics and Engineering, 2018, 332: 47-62

35. Anitescu C, Atroshchenko E, Alajlan N, et al. Artificial neural network methods for the solution of second order boundary value problems. Computers, Materials \& Continua, 2019, 59(1): 345-359

36. Xu J H, Feng X Q, Cen J, et al. Precision forward design for 3D printing using kinematic sensitivity via Jacobian matrix considering uncertainty. The International Journal of Advanced Manufacturing Technology, 2020, 110(11): 3257-3271

37. Xu J H, Wang K, Gao M Y, et al. Biomechanical performance design of joint prosthesis for medical rehabilitation via generative structure optimization. Computer Methods in Biomechanics and Biomedical Engineering, 2020, 23(15): 1163-1179

38. Xu J H, Wang K, Sheng H S, et al. Energy efficiency optimization for ecological $3 \mathrm{D}$ printing based on adaptive multi-layer customization. Journal of Cleaner Production, 2020, 245: 118826

39. Chen $\mathrm{C}$ T. Extensions of the TOPSIS for group decision-making under fuzzy environment. Fuzzy Sets and Systems, 2000, 114(1): $1-9$

40. Sun K K, Qiu J B, Karimi H R, et al. A novel finite-time control for nonstrict feedback saturated nonlinear systems with tracking error constraint. IEEE Transactions on Systems, Man, and Cybernetics. Systems, 2020 (in press)

41. Saaty T L. How to make a decision: The analytic hierarchy process. Interfaces, 1994, 24(6): 19-43

42. Yang X L, Ding J H, Hou H. Application of a triangular fuzzy AHP approach for flood risk evaluation and response measures analysis. Natural Hazards, 2013, 68(2): 657-674

43. Seresht N G, Fayek A R. Computational method for fuzzy arithmetic operations on triangular fuzzy numbers by extension principle. International Journal of Approximate Reasoning, 2019, 106: 172193 